\title{
A real triplet-singlet extended Standard Model: dark matter and collider phenomenology
}

\author{
Nicole F. Bell, ${ }^{a}$ Matthew J. Dolan, ${ }^{a}$ Leon S. Friedrich, ${ }^{a, 1}$ \\ Michael J. Ramsey-Musolf ${ }^{b, c, d}$ and Raymond R. Volkas ${ }^{a}$ \\ ${ }^{a}$ ARC Centre of Excellence for Dark Matter Particle Physics, \\ School of Physics, The University of Melbourne, \\ Victoria 3010, Australia \\ ${ }^{b}$ Tsung-Dao Lee Institute and School of Physics and Astronomy, \\ Shanghai Jiao Tong University, \\ 800 Dongchuan Road, Shanghai, 200240, China \\ ${ }^{c}$ Amherst Center for Fundamental Interactions, \\ Department of Physics, University of Massachusetts Amherst, \\ Amherst, MA 01003, U.S.A. \\ ${ }^{d}$ Kellogg Radiation Laboratory, California Institute of Technology, \\ Pasadena, CA 91125, U.S.A. \\ E-mail: n.bell@unimelb.edu.au, matthew.dolan@unimelb.edu.au, \\ lfriedrich@student . unimelb.edu.au, mjrm@physics.umass.edu, \\ raymondv@unimelb.edu.au
}

ABstract: We examine the collider and dark matter phenomenology of the Standard Model extended by a hypercharge-zero $\mathrm{SU}(2)$ triplet scalar and gauge singlet scalar. In particular, we study the scenario where the singlet and triplet are both charged under a single $\mathbb{Z}_{2}$ symmetry. We find that such an extension is capable of generating the observed dark matter density, while also modifying the collider phenomenology such that the lower bound on the mass of the triplet is smaller than in minimal triplet scalar extensions to the Standard Model. A high triplet mass is in tension with the parameter space that leads to novel electroweak phase transitions in the early universe. Therefore, the lower triplet masses that are permitted in this extended model are of particular importance for the prospects of successful electroweak baryogenesis and the generation of gravitational waves from early universe phase transitions.

Keywords: Beyond Standard Model, Higgs Physics

ArXiv EPrint: 2010.13376

\footnotetext{
${ }^{1}$ Corresponding author.
} 


\section{Contents}

1 Introduction 1

2 Model 3

2.1 Type-I, $\mathbb{Z}_{2}: S \rightarrow S, \Sigma \rightarrow-\Sigma$

2.2 Type-II, $\mathbb{Z}_{2}: S \rightarrow-S, \Sigma \rightarrow \Sigma$

2.3 Type-III, $\mathbb{Z}_{2}: S \rightarrow S, \Sigma \rightarrow \Sigma$

2.4 Type-IV, $\mathbb{Z}_{2}: S \rightarrow-S, \Sigma \rightarrow-\Sigma$

2.4.1 Mass matrix and mixing angles $\quad 7$

2.4.2 Oblique corrections 8

2.4.3 Parameter selection and coupling constraints 9

3 Dark matter phenomenology 10

4 Collider phenomenology 15

$\begin{array}{lll}4.1 & \text { Decays } & 17\end{array}$

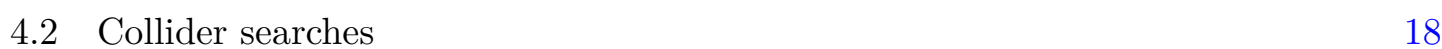

4.2.1 Small mass difference, $\Delta m<50 \mathrm{GeV} \quad 19$

4.2.2 Large mass difference, $\Delta m>m_{H} \quad 20$

4.2.3 Intermediate mass difference, $50 \mathrm{GeV}<\Delta m<m_{H} \quad 21$

$\begin{array}{lll}4.3 & \text { Alternative searches and future prospects } & 21\end{array}$

5 Conclusion $\quad 22$

A Loop induced annihilation into monochromatic photons 23

\section{Introduction}

The prospect of a strongly first order electroweak phase transition (EWPT) is of great interest due to its potential to generate the observed baryon asymmetry via electroweak baryogenesis (EWBG), and the prospect of such a transition resulting in a detectable gravitational wave background. For reviews on EWBG, phase transition induced gravitational waves, and the EWPT in general, see refs. [1, 2], [3, 4], and [4, 5], respectively. However, non-perturbative lattice studies show that instead of featuring a first order transition, the Standard Model (SM) EWPT proceeds via a crossover transition [6-11]. Therefore, the SM can neither generate the observed baryon asymmetry, nor produce detectable gravitational waves during the EWPT.

However, if there is new physics that couples to the SM Higgs boson, the phase transition may instead be first order. This is often achieved by introducing gauge singlet 
scalars [12-55]. Scalar singlet extensions are capable of generating the desired phase transition and have the benefit of being weakly constrained at colliders due to their small production cross sections.

Another class of models that can feature a strongly first order transition are those that introduce additional scalar SU(2) multiplets. This class of model generally features more complex phenomenology and faces more severe collider constraints. For example the extensively studied two-Higgs-doublet model [56-70], which is typically inspired by supersymmetry [71-80], and $\mathrm{SU}(2)$ triplet scalar extension models [81-89] fall into this category. The addition of a triplet scalar is the simplest scalar $\mathrm{SU}(2)$ multiplet extension in the sense that it has the fewest additional physical particles, and the fewest new parameters present without imposing additional symmetries.

$\mathrm{SU}(2)$ triplet scalars have been studied extensively in the context of dark matter (DM) models [86, 88, 90-97], their contribution to the EWPT [83, 85, 89, 98], and their collider phenomenology [81, 87, 88, 99-101]. Unlike singlet scalars, they will always be produced at colliders via charged and neutral current Drell-Yan processes, independent of their coupling or mixing with the SM Higgs. Reference [88] examines the present and future bounds that apply if the neutral component of the triplet is stable. They find that recent disappearing charged track searches using $36 \mathrm{fb}^{-1}$ of data require the triplets to have masses larger than about $287 \mathrm{GeV}$. If the neutral triplet were unstable, then the lower bound on the mass decreases to around $230 \mathrm{GeV}$ [87]. Assuming no detection of new physics, this lower bound will increase with the inclusion of more up-to-date analyses utilising more data. Eventually this lower bound will be in tension with the parameter space required for a novel EWPT. Furthermore, while the SU(2) triplet scalar may be stable and contribute to the DM density, in the parameter space relevant to a novel EWPT the triplet will only ever contribute a small fraction of the observed DM density.

However, these collider and DM constraints are obviously only applicable in pure triplet scalar extensions. The triplet may simply be one of several additional particles in more complex models. For example, consider real triplet scalars in the Georgi-Machacek model [102-105], extended supersymmetric models [106], or those arising from the breaking of some GUT symmetry, e.g., from the $\mathbf{2 1 0}$ of $\mathbf{S O}(10)$ [107]. Assuming that at least some of this additional particle content is light, the triplet's collider and DM phenomenology may differ significantly from the minimal model.

In this paper, we investigate how the introduction of a gauge singlet scalar modifies the collider and DM phenomenology of the $\mathrm{SU}(2)$ triplet scalar extended SM. We focus on a subset of models with this particle content where both of the new scalars are charged under a single $\mathbb{Z}_{2}$ symmetry and neither scalar gains a vacuum expectation value (VEV) at zero temperature. We find that such an extension is capable of relaxing the lower bound on the mass of the $\mathrm{SU}(2)$ triplet, as is desirable for a novel EWPT, while also enabling the production of the correct DM relic density.

The combination of hypercharge-zero $\mathrm{SU}(2)$ triplet and gauge singlet scalar extensions has been considered previously in refs. [93, 95, 106, 108, 109]. Reference [106] examines the phenomenology of a singlet and triplet extended supersymmetric model, where both of the new scalars can gain VEVs at zero temperature. The phenomenology of the model 
considered in ref. [106] differs significantly from the model considered here as we are not imposing supersymmetry, have only a single Higgs doublet, and none of our scalars gain a zero temperature VEV. Reference [108] considers a singlet-triplet extended two Higgs doublet model in the context of EWBG, but examines neither the DM constraint nor collider constraints due to the production of the new scalars. Once again, the second Higgs doublet prevents a direct analogy. However, some of the collider physics we consider in this work may also be applicable, particularly if the additional Higgs doublet components do not significantly modify the production and decay mechanisms of the singlet and triplet scalars. References $[93,109]$ examine a model with the same particle content that we consider here. However, they consider a subset of the parameter space where an additional $\mathbb{Z}_{2}$ symmetry results in both the singlet and neutral component of the $\mathrm{SU}(2)$ triplet being stable and, therefore, contributing to the DM density. As a result, their study of this two-component DM model focuses on a complementary region of parameter-space that features very different collider and DM phenomenology. Reference [95] considers the same model that we examine here, with a focus on the dark matter phenomenology. We extend this previous work with a more detailed discussion of the collider phenomenology, updated direct detection constraints, and the inclusion of indirect detection and electroweak precision constraints.

The remainder of this paper is structured as follows. In section 2 we define our model, motivate the study of a specific $\mathbb{Z}_{2}$ symmetric sub-model, and outline our scalar coupling constraints and parameterisation. We then examine the dark matter and collider phenomenology of our model in sections 3 and 4, respectively. Finally, we conclude in section 5 .

\section{$2 \quad$ Model}

We extend the Standard Model by adding both a real scalar singlet $S$, and a real scalar field $\Sigma$ transforming as $(1,3,0)$ under the $\mathrm{SU}(3) \times \mathrm{SU}(2) \times \mathrm{U}(1)_{Y} \mathrm{SM}$ gauge group. We refer to this model as the $\Sigma x \mathrm{SM}$. We consider the most general renormalisable scalar potential,

$$
\begin{aligned}
V_{\Sigma x \mathrm{SM}}= & -\mu_{H}^{2} H^{\dagger} H-\frac{1}{2} \mu_{\Sigma}^{2} \operatorname{Tr}\left(\Sigma^{2}\right)-\frac{1}{2} \mu_{S}^{2} S^{2} \\
& +\lambda_{H}\left(H^{\dagger} H\right)^{2}+\frac{1}{4} \lambda_{\Sigma} \operatorname{Tr}\left(\Sigma^{2}\right)^{2}+\frac{1}{4} \lambda_{S} S^{4} \\
& +\frac{1}{\sqrt{2}} a_{H \Sigma} H^{\dagger} \Sigma H+a_{H S} H^{\dagger} H S+\frac{1}{2} a_{\Sigma S} \operatorname{Tr}\left(\Sigma^{2}\right) S+\frac{1}{3} a_{S} S^{3} \\
& +\frac{1}{2} \lambda_{H \Sigma} \operatorname{Tr}\left(\Sigma^{2}\right) H^{\dagger} H+\frac{1}{2} \lambda_{H S} H^{\dagger} H S^{2}+\frac{1}{4} \lambda_{\Sigma S} \operatorname{Tr}\left(\Sigma^{2}\right) S^{2} \\
& +\frac{1}{\sqrt{2}} \lambda_{H \Sigma S} H^{\dagger} \Sigma H S+b S,
\end{aligned}
$$

where $H$ is the SM Higgs doublet, and we use the notation

$$
\Sigma=\left[\begin{array}{cc}
\frac{1}{\sqrt{2}} \Sigma^{0} & \Sigma^{+} \\
\Sigma^{-} & -\frac{1}{\sqrt{2}} \Sigma^{0}
\end{array}\right], \quad H=\left[\begin{array}{c}
H^{+} \\
\frac{1}{\sqrt{2}}\left(H^{0}+i A^{0}\right)
\end{array}\right] .
$$




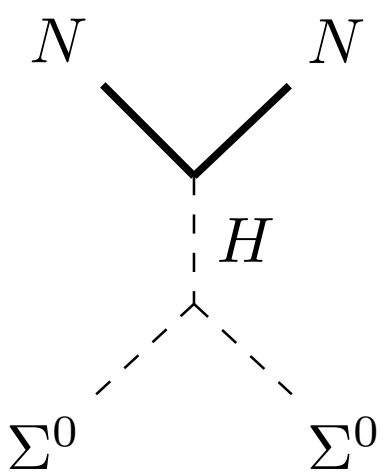

(a)

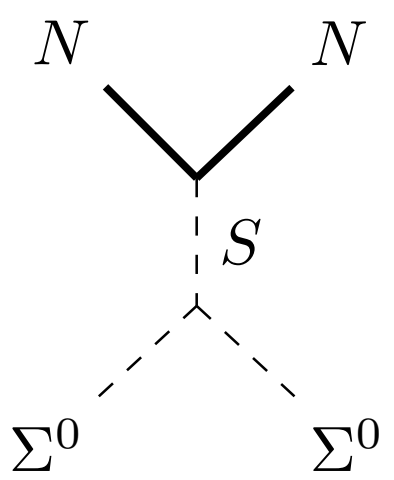

(b)

Figure 1. DM-nucleus scattering diagrams for the type-I $\Sigma x \mathrm{SM}$, including; (a) the SM Higgs mediated scattering present in the $\Sigma \mathrm{SM}$, and (b) a singlet scalar mediated scattering, which is dependent the singlet mixing with the SM Higgs. The nucleus is represented by $N$, the dark matter by $\Sigma^{0}$.

This Lagrangian has many parameters and can result in widely different phenomenology depending on what values the couplings take and whether any are disallowed by additional symmetries. By introducing a $\mathbb{Z}_{2}$ symmetry under which just $S$ and $\Sigma$ transform, this model can be categorised into four types based on the $\mathbb{Z}_{2}$ charge assignments. The following sections will provide an overview of the expected phenomenology of each type of model. The remainder of the paper will focus on the fourth type that we consider, which is outlined last in section 2.4, as it has the most phenomenological promise from the perspective of achieving EWBG.

\section{$2.1 \quad$ Type-I, $\mathbb{Z}_{2}: S \rightarrow S, \Sigma \rightarrow-\Sigma$}

In this scenario, only the triplet is charged under a $\mathbb{Z}_{2}$ symmetry. This results in a stable triplet, such that $\Sigma^{0}$ contributes to the DM density. The singlet will, in general, mix with the SM Higgs through the $a_{H S}$ and $\lambda_{H S}$ couplings.

In the absence of the singlet, this scenario corresponds to the pure $\Sigma$ extended SM $(\Sigma \mathrm{SM})$ with a stable triplet. As is discussed in refs. [87, 88], the parameter space for a light stable triplet is strongly constrained by dark matter direct detection experiments. However, the introduction of the singlet provides an additional annihilation channel for the triplets in the early universe, thus reducing their relic density. Furthermore, the singlet can reduce the direct detection scattering cross section. In the $\Sigma$ SM the DM-nucleus scattering is dominated by a Higgs-exchange diagram, shown in figure 1a, which is now complemented by a singlet-exchange diagram, shown in figure 1b. There may be regions of the parameter space where these two scattering diagrams partially cancel, thus reducing the direct detection scattering rate. The combination of these two factors may open up some of the previously excluded parameter space of triplet couplings.

The constraints on the singlet scalar couplings will be similar to those appearing in the real singlet scalar extended standard model, which has already been studied extensively in the context of electroweak phase transitions [12-55]. The constraints may differ slightly 
due to the possibility of $S \rightarrow \Sigma \Sigma$ decays and new collider production diagrams involving the triplets. Nonetheless, we expect the singlet to be relatively unconstrained, provided we enforce $m_{S} \gtrsim m_{H} / 2$ to avoid the decay $H \rightarrow S S$.

However, as discussed in refs. [81, 87, 88], there is also a disappearing charged track constraint which currently requires $m_{\Sigma} \gtrsim 287 \mathrm{GeV}$. This constraint is not affected by the singlet scalar, and thus will exclude most of the parameter space of interest to us. Therefore we will not examine the Type-I $\Sigma x$ SM further in this paper.

\subsection{Type-II, $\mathbb{Z}_{2}: S \rightarrow-S, \Sigma \rightarrow \Sigma$}

In this $\Sigma x \mathrm{SM}$ variant, only the singlet is charged under a $\mathbb{Z}_{2}$ symmetry. Thus the singlet will be stable and contribute to the DM density, while the triplet will mix with the SM Higgs due to the $a_{H \Sigma}$ coupling, though this mixing must be small due to $\rho$-parameter constraints.

Minimal singlet scalar DM models are strongly constrained by competing requirements on the singlet's couplings with the SM Higgs. On one hand, this coupling has to be small as the DM direct detection scattering is mediated by the SM Higgs. On the other, the coupling has to be large enough to allow the singlet to annihilate in the early universe, in order to avoid an over-density of dark matter. There is an allowed region of the singlet scalar parameter space near $m_{S} \sim m_{H} / 2$, due to a resonance of the DM annihilation rate, but otherwise, most of the parameter space is excluded (see refs. [110, 111] and references therein). These constraints may be relaxed significantly by the presence of the triplet, as it both opens up a new annihilation channel that is not directly tied to the direct detection scattering rate $(S S \rightarrow \Sigma \Sigma \rightarrow \mathrm{SM}$ ), and the triplet may act to reduce the direct detection scattering rate in a manner analogous to the type-I scenario.

The collider phenomenology of the triplet will be similar to the $\Sigma$ SM phenomenology that has already been discussed extensively in refs. [81, 87, 99-101]. The main effect of the singlet is the introduction of the $\Sigma^{0} \rightarrow S S$ decay channel. Assuming this decay is kinematically allowed, this will lead to events with large missing energy, which some SUSY chargino-neutralino searches are sensitive to. However, this branching ratio can be made arbitrarily small by tuning the scalar couplings or by having more massive singlets. For intermediate values of the branching ratio the effect of the new missing energy signal region will be to siphon events away from other signal regions.

A preliminary examination of collider constraints for this $\Sigma x \mathrm{SM}$ type was carried out using the same methodology used in ref. [87]. We found that the decrease in events in some signal regions was compensated for by the gain of events in signal regions with large missing energy, leading to no significant reduction in the lower bound on the triplet mass.

Therefore, while this type of model may be able to satisfy DM constraints, most of the parameter space of interest to us is still excluded. Therefore we will not examine this type of $\Sigma x \mathrm{SM}$ model any further.

\subsection{Type-III, $\mathbb{Z}_{2}: S \rightarrow S, \Sigma \rightarrow \Sigma$}

In this scenario, there is no $\mathbb{Z}_{2}$ symmetry and all couplings are allowed, leading to a very large number of free parameters. In general, these couplings will lead to mixing between 
all of the scalars. The scalar mixing will result in all the new scalars being unstable, such that there is no DM candidate.

While the singlet-triplet mixing may be very large, the mixing with the SM Higgs is constrained by the $\rho$-parameter and Higgs coupling measurements. Therefore, the dominant production mechanism at colliders will likely still be pair production via charged and neutral current Drell-Yan processes, as in the $\Sigma$ SM. However in addition to decaying into fermion or weak gauge boson pairs, the new scalars may also decay into one another. For example, assuming $m_{S^{\prime}}$ is less than $m_{\Sigma^{ \pm^{\prime}}}$ and $m_{\Sigma^{0^{\prime}}}$, we have two additional decays,

- $\Sigma^{0^{\prime}} \rightarrow S^{\prime(*)} S^{\prime}$,

- $\Sigma^{ \pm^{\prime}} \rightarrow W^{ \pm(*)} S^{\prime}$

where we have used primed indices to denote mass eigenstates whose primary component is the corresponding unprimed particle. Each of the scalars produced in such a decay would subsequently decay into fermion or weak gauge boson pairs. The most constraining current analyses for the $\Sigma \mathrm{SM}$ come about from multilepton searches [87]. These longer scalar decay chains increase the likelihood of pair production events leading to a multilepton final state, which results in more severe constraints. This may be partly offset by the lower energies of the final state particles in these longer decay chains, leading to lower efficiency cuts in the relevant analyses.

Without a more in-depth analysis of the collider phenomenology, it is not clear whether or not this $\Sigma x \mathrm{SM}$ model type can reduce the lower bound on the triplet mass. Consequently, the parameter space available for novel electroweak phase transitions may still be severely restricted. Additionally, the type-III model has no dark matter candidate. For these reasons, together with the complication of a large number of phenomenologically relevant parameters, we defer examination of this model to future work, and will instead focus on the fourth and final type.

\subsection{Type-IV, $\mathbb{Z}_{2}: S \rightarrow-S, \Sigma \rightarrow-\Sigma$}

In this model type both the singlet and triplet are charged under a $\mathbb{Z}_{2}$ symmetry. Thus one or both particles will be stable and contribute to the dark matter relic density. This scenario can be further broken up into two sub-categories depending on whether the new scalars are charged under the same or two different $\mathbb{Z}_{2}$ symmetries.

If $\lambda_{H \Sigma S}=0$, which effectively corresponds to there being two separate $\mathbb{Z}_{2}$ symmetries, then the neutral component of both the triplet and the singlet are stable and both will contribute to the DM relic density. It is likely that this scenario will face the same severe constraints that are encountered in minimal triplet DM model with $\mu_{\Sigma}^{2}>0$. In principle the couplings with the new singlets may reduce the triplet DM density somewhat via $\Sigma \Sigma \rightarrow S S$ annihilation, and this scenario may be worth examining. However, the constraints from disappearing charged tracks, as discussed in section 2.1, will still apply. Thus, a low-mass triplet would still be excluded in this scenario. Note that this sub-type with $\lambda_{H \Sigma S}=0$ is the same model as the two-component DM model considered in ref. [109]. However, 
they allow for $\mu_{\Sigma}^{2}<0$, and thus allow the triplet component of the DM to avoid direct detection constraints.

If instead both scalars are charged under the same $\mathbb{Z}_{2}$ symmetry, which allows $\lambda_{H \Sigma S} \neq 0$, then after electroweak symmetry breaking the $\lambda_{H \Sigma S}$ term will induce mixing between the singlet and neutral component of the triplet. Therefore, if the singlet-like mass eigenstate is lighter than the triplet-like one, then the DM will only consist of singlet-like particles, allowing us to avoid the severe triplet DM direct detection constraints that are present when $\mu_{\Sigma}^{2}<0$.

The triplets can still be produced at colliders as in the $\Sigma \mathrm{SM}$, via charged and neutral current Drell-Yan processes. However, unlike the $\Sigma \mathrm{SM}$, the $\lambda_{H \Sigma S}$ mixing term allows the triplets to decay rapidly into the neutral DM candidate and SM particles. This leads to events with large missing energy and removes the disappearing charged track constraint.

This model has the prospect of both alleviating collider constraints on triplet scalars, thus increasing the parameter space available for novel electroweak phase transitions, and presenting a viable dark matter candidate. Therefore, it is this version of the $\Sigma x \mathrm{SM}$ that we will study for the remainder of this paper.

\subsubsection{Mass matrix and mixing angles}

After electroweak symmetry breaking, the $\lambda_{H \Sigma S}$ term will lead to a mass term that will cause mixing between the singlet scalar and neutral component of the triplet,

$$
\begin{aligned}
V_{\Sigma x \mathrm{SM}} & \supset \frac{1}{2}\left(\begin{array}{ll}
\Sigma^{0} & S
\end{array}\right)\left(\begin{array}{cc}
-\mu_{\Sigma}^{2}+\frac{1}{2} v_{H}^{2} \lambda_{H \Sigma} & -\frac{1}{4} v_{H}^{2} \lambda_{H \Sigma S} \\
-\frac{1}{4} v_{H}^{2} \lambda_{H \Sigma S} & -\mu_{S}^{2}+\frac{1}{2} v_{H}^{2} \lambda_{H S}
\end{array}\right)\left(\begin{array}{c}
\Sigma^{0} \\
S
\end{array}\right), \\
& =\frac{1}{2}\left(\begin{array}{ll}
\Sigma^{0^{\prime}} & S^{\prime}
\end{array}\right)\left(\begin{array}{cc}
m_{\Sigma^{0^{\prime}}}^{2} & 0 \\
0 & m_{S^{\prime}}^{2}
\end{array}\right)\left(\begin{array}{c}
\Sigma^{0^{\prime}} \\
S^{\prime}
\end{array}\right)
\end{aligned}
$$

where we have introduced the mass basis,

$$
\left(\begin{array}{c}
\Sigma^{0^{\prime}} \\
S^{\prime}
\end{array}\right)=\left(\begin{array}{cc}
\cos \theta_{S} & \sin \theta_{S} \\
-\sin \theta_{S} & \cos \theta_{S}
\end{array}\right)\left(\begin{array}{c}
\Sigma^{0} \\
S
\end{array}\right)
$$

We choose to define the scalar mixing angle $\theta_{S}$ such that $\sin ^{2} \theta_{S} \leq 0.5$, in order to ensure that the mass eigenstate labelled $\Sigma^{0^{\prime}}$ always consists primarily of $\Sigma^{0}$. As will be discussed in subsequent sections, the collider and DM phenomenology is very sensitive to the neutral scalar mass difference. As it appears frequently in the discussion, we introduce the notation

$$
\Delta m=m_{\Sigma^{0^{\prime}}}-m_{S^{\prime}} .
$$

The tree-level mass of the $\Sigma^{+}$is simply given by the first diagonal element of the neutral scalar mixing matrix in eq. (2.3a). In the absence of mixing, $\lambda_{H \Sigma S}=0$, the masses of the charged and neutral components of the triplet would be degenerate. However, once the SM Higgs breaks the $\mathrm{SU}(2)$ symmetry and gives masses to the $W$ and $Z$ bosons, electroweak radiative corrections to the triplet mass lead to a small mass splitting of about $166 \mathrm{MeV}$ [90]. For this initial study we neglect the effect that the singlet has on the 


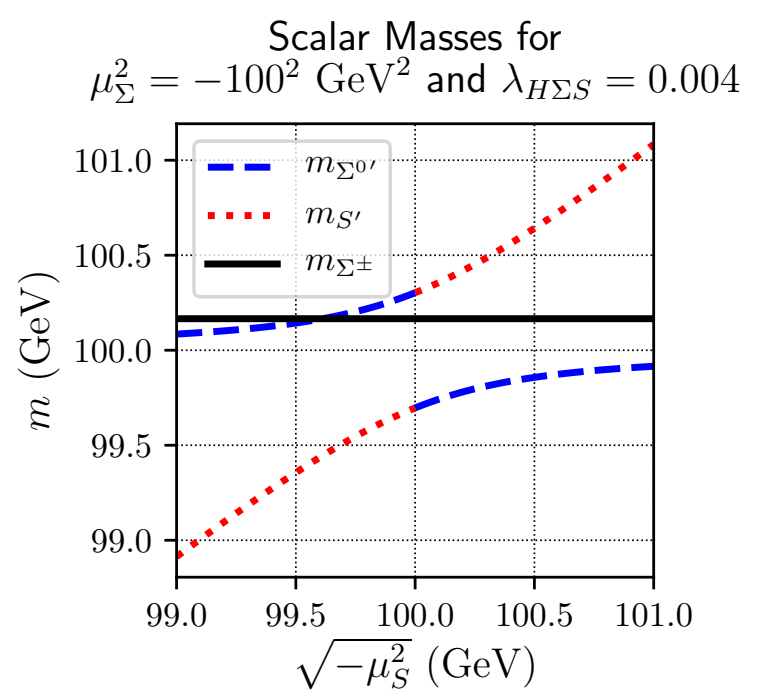

Figure 2. Masses of the new scalars as a function of $\mu_{S}^{2}$. The solid black, dashed blue, and dotted green lines give the masses of the $\Sigma^{ \pm}, \Sigma^{0^{\prime}}$, and $S^{\prime}$ particles, respectively. All other scalar couplings were set to zero. The identification of $\Sigma^{0^{\prime}}$ as the mass eigenstate that consists primarily of $\Sigma^{0}$ leads to a discontinuity in the labelling of the mass eigenstates when $\mu_{S}^{2}=-100^{2} \mathrm{GeV}^{2}$.

radiative mass correction, which may be significant for large mixing angles, and instead approximate this splitting by setting.

$$
m_{\Sigma^{+}}=\sqrt{-\mu_{\Sigma}^{2}+\frac{1}{2} v_{H}^{2} \lambda_{H \Sigma}}+166 \mathrm{MeV} .
$$

In minimal triplet models, this radiative mass splitting always leads to the charged component of the triplet being more massive than the neutral one. This is not the case in our model as the $\mathrm{SU}(2)$ symmetry breaking neutral scalar mixing can raise the mass of the $\Sigma^{0^{\prime}}$. Figure 2 illustrates this by showing how the masses of the scalars behave as a function of $\mu_{S}^{2}$ for some benchmark parameters. The small range for $\mu_{S}^{2}$ and small value for $\lambda_{H \Sigma S}$ in figure 2 were chosen such that the $166 \mathrm{MeV}$ radiative mass splitting is clearly visible. Note that the convention of using $\Sigma^{0^{\prime}}$ to denote the mass eigenstate that consists primarily of $\Sigma^{0}$ leads to a discontinuity in the labelling of the neutral scalar masses and couplings. This discontinuity in the labelling is clearly visible in figure 2 .

\subsubsection{Oblique corrections}

The singlet and triplet scalars will result in new electroweak radiative corrections, which are constrained by electroweak precision data. These corrections can be parameterised by the oblique parameters. The current limits on these parameters are [112]

$$
\begin{aligned}
& \Delta S=S-S_{\mathrm{SM}}=-0.01 \pm 0.10, \\
& \Delta T=T-T_{\mathrm{SM}}=0.03 \pm 0.12, \\
& \Delta U=U-U_{\mathrm{SM}}=0.02 \pm 0.11 .
\end{aligned}
$$

Our new particle content will contribute to the $T$ and $U$ parameters via one-loop diagrams. There is no contribution to the $S$ parameter as we have introduced neither new 
particles with hypercharge nor new particles that mix with the SM Higgs. The relevant loop diagrams and resulting loop functions also appear in the minimal $\mathrm{SU}(2)$ triplet extension. This allows us to write our contributions as,

$$
\begin{aligned}
& T_{\Sigma \mathrm{XSM}}=\cos ^{2} \theta_{S} T_{\Sigma \mathrm{SM}}\left(m_{\Sigma^{0^{\prime}}}\right)+\sin ^{2} \theta_{S} T_{\Sigma \mathrm{SM}}\left(m_{S^{\prime}}\right), \\
& U_{\Sigma \mathrm{xSM}}=\cos ^{2} \theta_{S} U_{\Sigma \mathrm{SM}}\left(m_{\Sigma^{0^{\prime}}}\right)+\sin ^{2} \theta_{S} U_{\Sigma \mathrm{SM}}\left(m_{S^{\prime}}\right),
\end{aligned}
$$

where $T_{\Sigma \mathrm{SM}}, U_{\Sigma \mathrm{SM}}$ are the leading order one-loop contributions in the minimal triplet extension. ${ }^{1}$ These functions can be found in refs. [81, 100, 113], and are well approximated by,

$$
\begin{aligned}
T_{\Sigma \mathrm{SM}}(m) & \approx \frac{1}{6 \pi} \frac{1}{s_{W}^{2} c_{W}^{2}} \frac{\left(m-m_{\Sigma^{+}}\right)^{2}}{m_{Z}^{2}}, \\
U_{\Sigma \mathrm{SM}}(m) & \approx \frac{m-m_{\Sigma^{+}}}{3 \pi m_{\Sigma^{+}}} .
\end{aligned}
$$

These corrections can be significant if the $\lambda_{H \Sigma S}$ coupling is large, such that there is a large mixing angle and triplet-component mass differences $\left(m_{\Sigma^{0^{\prime}}}-m_{\Sigma^{+}}\right)$.

In order to quantify the constraints coming from electroweak precision observables, we take the same approach used in refs. $[18,33,43,51]$ and define

$$
\begin{aligned}
\Delta \chi^{2} & =\sum_{i, j}\left(\mathcal{O}_{i, \Sigma \mathrm{xSM}}-\Delta \mathcal{O}_{i}^{0}\right)\left(\sigma^{2}\right)_{i j}^{-1}\left(\mathcal{O}_{j, \Sigma \mathrm{xSM}}-\Delta \mathcal{O}_{j}^{0}\right) \\
\sigma_{i j}^{2} & =\sigma_{i} \rho_{i j} \sigma_{j}
\end{aligned}
$$

where $\mathcal{O}_{i} \in\{S, T, U\}, \Delta \mathcal{O}_{i}^{0}$ and $\sigma_{i}$ denote the central values and errors in eqs. (2.7), and the correlation matrix is [112],

$$
\rho_{i j}=\left(\begin{array}{ccc}
1 & 0.92 & -0.8 \\
0.92 & 1 & -0.93 \\
-0.8 & -0.93 & 1
\end{array}\right) .
$$

We then consider any points with $\Delta \chi^{2}>7.82$, which corresponds to the $95 \%$ C.L. for three degrees of freedom, to be excluded by electroweak precision observables.

\subsubsection{Parameter selection and coupling constraints}

Aside from the ordinary SM Higgs couplings, which are fixed by requiring $m_{H}=125 \mathrm{GeV}$ and $v_{H}=246 \mathrm{GeV}$, there are eight free scalar potential parameters in eq. (2.1). However, given that the scalar masses are more phenomenologically relevant, we will instead parameterise our model in terms of the following eight parameters; $m_{S^{\prime}}, m_{\Sigma^{0^{\prime}}}, \lambda_{\Sigma S}, \mu_{\Sigma}^{2}, \lambda_{H S}$, $\lambda_{\Sigma}, \lambda_{S}$ and $\lambda_{H \Sigma S}$. The first five of these parameters fix all of the components of the mass matrix, eq. (2.3a), and thus uniquely determine $\mu_{S}^{2}, \lambda_{H \Sigma}, m_{\Sigma^{ \pm}}$, and $\theta_{S}$. However, note that

\footnotetext{
${ }^{1}$ The minimal triplet model with a non-zero triplet VEV and triplet-Higgs mixing will have tree level and additional one-loop contributions that are not present in our model.
} 
the eight parameters are not entirely independent, as some combinations will be unphysical. This can be seen by noting that $m_{\Sigma^{0^{\prime}}}=m_{S^{\prime}}$ requires $\lambda_{H \Sigma S}=0$, such that a non-zero selection for $\lambda_{H \Sigma S}$ would be unphysical. Particularly, physical choices must satisfy,

$$
\left|m_{\Sigma^{0^{\prime}}}^{2}-m_{S^{\prime}}^{2}\right| \geq \frac{1}{2} v_{H}^{2} \lambda_{H \Sigma S} .
$$

We also require that the scalar potential is bounded from below. In the absence of the $\lambda_{H \Sigma S}$ coupling, this requirement can be expressed as a simple set of inequalities. However, with non-zero $\lambda_{H \Sigma S}$, the full set of conditions are quite complicated. Vacuum stability conditions are derived in ref. [114] for several different models, including one with two real scalar singlets and a SM Higgs doublet. The constraints that apply to that model are also applicable to our model. Additionally, ref. [114] conveniently provides a supplementary Mathematica notebook that includes the necessary inequalities. Therefore, we will simply require that our couplings satisfy the relevant inequalities, eq. (61) in ref. [114], but we will not replicate them here.

We also require that our scalar couplings are perturbative. This requirement is generally more strict than simply requiring the couplings satisfy perturbative unitarity (see, e.g., refs. $[87,115,116])$. Given that $\lambda_{H \Sigma}$ is directly related to $m_{\Sigma^{+}}$via eq. (2.6), the perturbativity bound on $\lambda_{H \Sigma}$ can be translated into a mass bound for $m_{\Sigma^{+}}$. Therefore, motivated by the bound in ref. [87], we will ensure $\lambda_{H \Sigma}$ is perturbative by requiring $0<\mu_{\Sigma}^{2}<200^{2} \mathrm{GeV}^{2}$ and $m_{\Sigma^{+}}<400 \mathrm{GeV}$. For all other quartic scalar couplings $\lambda$, we will simply impose the requirement that $\lambda<2$.

Note that the presence of the triplet will modify the SM Higgs diphoton decay rate. This places constraints on $m_{\Sigma^{ \pm}}$and $\lambda_{H \Sigma}$. Given that this correction has already been discussed extensively in the literature, and that the correction is generally within three standard deviations of the measured value, we will not be discussing this constraint in detail and instead refer readers to refs. [87, 96, 100, 101, 108].

\section{Dark matter phenomenology}

In the limit where both $\lambda_{\Sigma S}$ and $\lambda_{H \Sigma S}$ are small, such that the singlet-triplet interactions are not significant, the DM phenomenology of our model will be very similar that of a minimal singlet or triplet scalar DM model. Thus, we may have to address the same issues faced by these models, which must somehow be resolved by the introduction of singlettriplet interactions. In particular, if $m_{\Sigma^{0^{\prime}}}<m_{S^{\prime}}$, such that the triplet-like neutral scalar $\Sigma^{0^{\prime}}$ is the DM candidate, one might expect to encounter the same issues that arise in minimal triplet scalar DM models:

- The triplet can rapidly annihilate into weak gauge bosons, such that the relic density of light triplets will only ever be a small fraction of the observed DM density.

- DM direct detection constraints require $\lambda_{H \Sigma}$ to be small, which generally requires $\mu_{\Sigma}^{2}<0$ and is incompatible with scenarios that feature novel electroweak phase transitions due to a triplet VEV in the early universe. 
Conversely, if $m_{S^{\prime}}<m_{\Sigma^{0^{\prime}}}$, such that the singlet-like neutral scalar $S^{\prime}$ is the DM candidate, one might expect to encounter the same issues that arise in minimal singlet scalar DM models:

- The singlet annihilates through its coupling with the SM Higgs, and thus requires $\lambda_{H S}$ to be large enough to avoid an over-density of dark matter.

- Simultaneously, $\lambda_{H S}$ must be small enough to avoid direct detection constraints

- Satisfying both of these conditions requires $m_{S} \sim m_{H} / 2$, such that the $S S \rightarrow H$ annihilation rate is resonantly enhanced.

The $\Sigma x$ SM may be capable of addressing some of these issues faced by the corresponding minimal DM models. In the case where $\Sigma^{0^{\prime}}$ is the DM candidate, a large neutral scalar mixing angle may reduce its coupling with the SM Higgs and weak gauge bosons. This would result in a both a smaller direct detection cross section and a smaller annihilation rate, and thus also a larger relic density. In the case where $S^{\prime}$ is the DM candidate, the $\Sigma x \mathrm{SM}$ features two new annihilation channels, $S^{\prime} S^{\prime} \rightarrow \Sigma^{\prime} \Sigma^{\prime} \rightarrow \mathrm{SM}$ and $S^{\prime} S^{\prime} \rightarrow W^{+} W^{-}$. These annihilation channels can keep the $S^{\prime}$ relic density small without requiring a large coupling with the SM Higgs.

To quantitatively examine the DM phenomenology of the $\Sigma x \mathrm{SM}$ we use MicrOMEGAS 5.0 .8 [117] to evaluate the relic density and direct detection scattering cross section. We use the observed dark matter density as measured by the Planck collaboration [118], $\Omega_{\mathrm{DM}} h^{2}=0.12$, and compare the cross section obtained by MicrOMEGAS to the XENON1T [119] 90\%-confidence upper bound on the spin-independent scattering cross section, after scaling to account for the fraction of the density of DM that is made up of our DM candidate. MicrOMEGAS also provides tools for the examination of indirect detection constraints arising from tree-level annihilation processes. We found that inclusion of DM indirect detection constraints arising from these annihilation processes did not lead to any additional constraints. However, MicrOMEGAS does not automatically evaluate general loop-induced annihilation processes such as $S^{\prime} S^{\prime} \rightarrow h^{*} \rightarrow \gamma \gamma$. This annihilation into monochromatic photons results in a very clean indirect detection signal. Therefore, we evaluate the diphoton annihilation cross section using the analytic formulae given in appendix A and compare this with the current Fermi-LAT limits [120].

We explore the $\Sigma x \mathrm{SM}$ parameter space by performing a random scan of the scalar potential parameters and evaluating the dark matter density and direct detection constraints for each point. We randomly select dimensionful parameters from a uniform distribution, with masses ranging from 65 to $300 \mathrm{GeV}$ and $\sqrt{\mu_{\Sigma}^{2}}$ ranging from 0 to $200 \mathrm{GeV}$. We randomly select the dimensionless quartic scalar couplings from a log-uniform distribution ranging from $10^{-4}$ to 2 . Note that the range of parameters does not include the $m_{S^{\prime}}, m_{\Sigma^{0^{\prime}}}<\frac{m_{H}}{2}$ region, as that region of parameter space requires one to avoid invisible Higgs decay constraints. This places severe constraints on the $\lambda_{H S}, \lambda_{H \Sigma}$, and $\lambda_{H \Sigma S}$ couplings. As one of the motivations for this model is the potential to generate a novel electroweak phase transition, we are interested in regions of parameter space free of these severe constraints on the scalar couplings. 


\section{Dark Matter Exclusion Scatterplot}
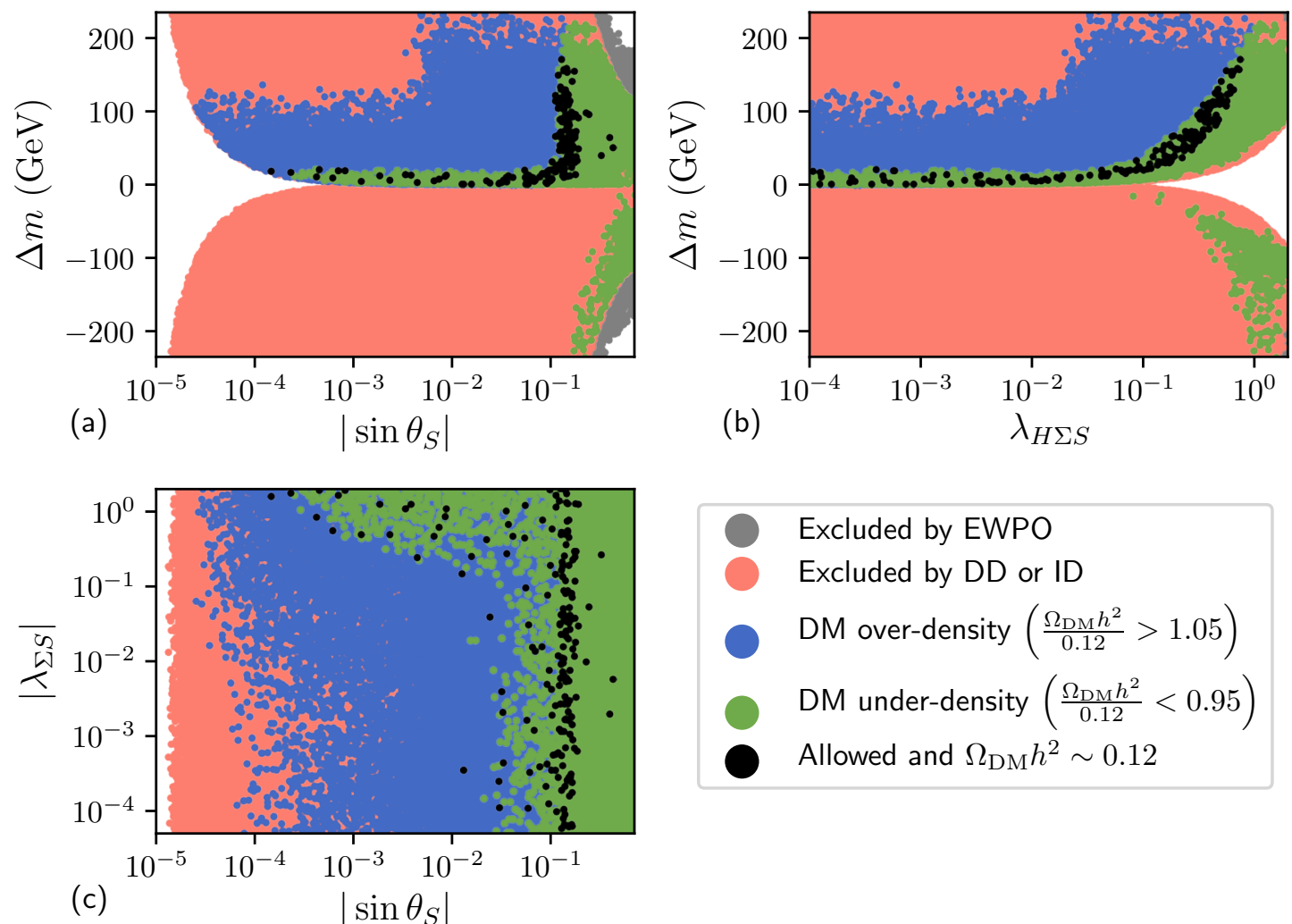

Figure 3. Scatter plots showing the dark matter constraints for a random scan of the parameter space. Grey points are excluded by electroweak precision observables (EWPO), red points are excluded by DM direct detection (DD) or DM indirect detection (ID), blue points are excluded by a DM over-density, green points are allowed but result in a DM under-density, and black points are allowed and approximately yield the correct relic density. As mentioned in the text, the indirect detection constraints are relatively weak and the red points are primarily excluded by direct detection.

Figure 3 shows a scatter plot of the DM results from the random scan of the parameter space. Grey points are excluded by electroweak precision observables (discussion in section 2.4.2), red points are excluded by direct or indirect detection, blue points are excluded by DM overproduction, green points are allowed but result in a DM under-density, and black points are allowed and approximately yield the correct relic density. For the parameter space considered here, the indirect detection constraints are subdominant. While several points are excluded by indirect detection, most of these are also excluded by either direct detection or a DM over-density. Only two points in the scan were excluded solely by indirect detection constraints. Examination of figure 3 reveals several key features:

1. Points with $\Delta m<0$ are excluded by DM direct detection unless $\lambda_{H \Sigma S}$, and thus also $\theta_{S}$, are large. This occurs because when $\Delta m<0$, such that $\Sigma^{0^{\prime}}$ is the DM candidate, our requirement that $\mu_{\Sigma}^{2}>0$ results in a large $\lambda_{H \Sigma}$ and large $\Sigma^{0^{\prime}}$ direct detection cross section. However, the constraint can be avoided if the $\theta_{S}$ mixing angle is large. This allows for cancellation in the $\Sigma^{0^{\prime}}-H$ quartic coupling, thus reducing the direct detection cross-section to acceptable levels. 
2. The points with $\Delta m<0$ allowed by direct detection do not reproduce the observed relic density. This occurs because the $\Sigma^{0^{\prime}}$ coupling to weak gauge bosons tends to result in a small relic density. When combined with the previous point, this means that $\Sigma^{0^{\prime}}$ cannot satisfy both the direct detection and relic density constraints when $\Delta m<0$.

3. The DM requirements are only satisfied when either $\left|\lambda_{\Sigma S}\right| \gtrsim 0.1$ or $\left|\sin \theta_{S}\right| \gtrsim 0.01$ (see black points in figure 3c). This occurs as the singlet tends to produce an over-density of dark matter, and in order to have the correct relic density it needs a sufficiently large annihilation cross-section. It cannot obtain this through couplings with the SM Higgs, ${ }^{2}$ as this would be in tension with direct detection constraints. Therefore it must annihilate either through its couplings with the triplet $\lambda_{\Sigma S}\left(S^{\prime} S^{\prime} \rightarrow \Sigma^{0^{\prime}} \Sigma^{0^{\prime}} \rightarrow \mathrm{SM}\right)$, or through its coupling to the weak gauge bosons, which it acquires through its mixing with the triplet, $\propto \sin \theta_{S}$. Therefore at least one of $\lambda_{\Sigma S}$ and $\theta_{S}$ must be large.

4. When $\left|\sin \theta_{S}\right| \lesssim 0.1$, the only points that satisfy all DM requirements are those with $0 \lesssim \Delta m \lesssim 30 \mathrm{GeV}$ (the thin horizontal strip of black points in figure 3a). As established in the previous point, obtaining the correct singlet relic density when $\left|\sin \theta_{S}\right| \lesssim 0.1$ relies on the $S^{\prime} S^{\prime} \rightarrow \Sigma^{0^{\prime}} \Sigma^{0^{\prime}} \rightarrow$ SM annihilation channel. This rate is kinematically suppressed when the singlet is much lighter than the triplet. Therefore, in order for this annihilation rate to be sufficiently large, $\Delta m=m_{\Sigma^{0^{\prime}}}-m_{S^{\prime}}$ cannot be too large. The precise value of the upper limit on $\Delta m$ depends on the maximum value of $\lambda_{\Sigma S}$ (we take $\left|\lambda_{\Sigma S}\right| \leq 2$ ).

5. Conversely, if $\left|\sin \theta_{S}\right| \gtrsim 0.1$, then the DM requirements can also be satisfied by a large $\Delta m$. This occurs because when $\theta_{S}$ is large, then the $S^{\prime}$ can annihilate into weak gauge bosons. This annihilation rate is not sensitive to $\Delta m$. In particular, when $\left|\sin \theta_{S}\right| \sim 0.1$, the $S^{\prime}$ can yield the correct relic density without relying on other annihilation channels.

From these observations we conclude that in order for the $\Sigma x \mathrm{SM}$ to provide a viable dark matter candidate, the scalar parameters generally fall into one of two categories:

(a) $\lambda_{\Sigma S}>0.1$ and $0<\Delta m<30 \mathrm{GeV}$.

(b) $\left|\sin \theta_{S}\right| \gtrsim 0.1, \lambda_{H \Sigma S} \gtrsim 0.1$, and $\Delta m>0$.

Aside from the requirements on $\Delta m$, the DM requirements can be satisfied by a wide range of scalar masses. To illustrate this, we select two sets of benchmark scalar couplings and show the mass dependence of the DM relic density and DM constraints in figures 4 and 5 , respectively.

The benchmark couplings used in figures $4 \mathrm{a}$ and 5 a correspond to the first category, where the correct relic density can only be obtained when $0<\Delta m \lesssim 30 \mathrm{GeV}$, or when

\footnotetext{
${ }^{2}$ Except for the region where there is a resonance $m_{S^{\prime}} \sim m_{H} / 2$ as discussed below.
} 


\section{Dark Matter Relic Density}
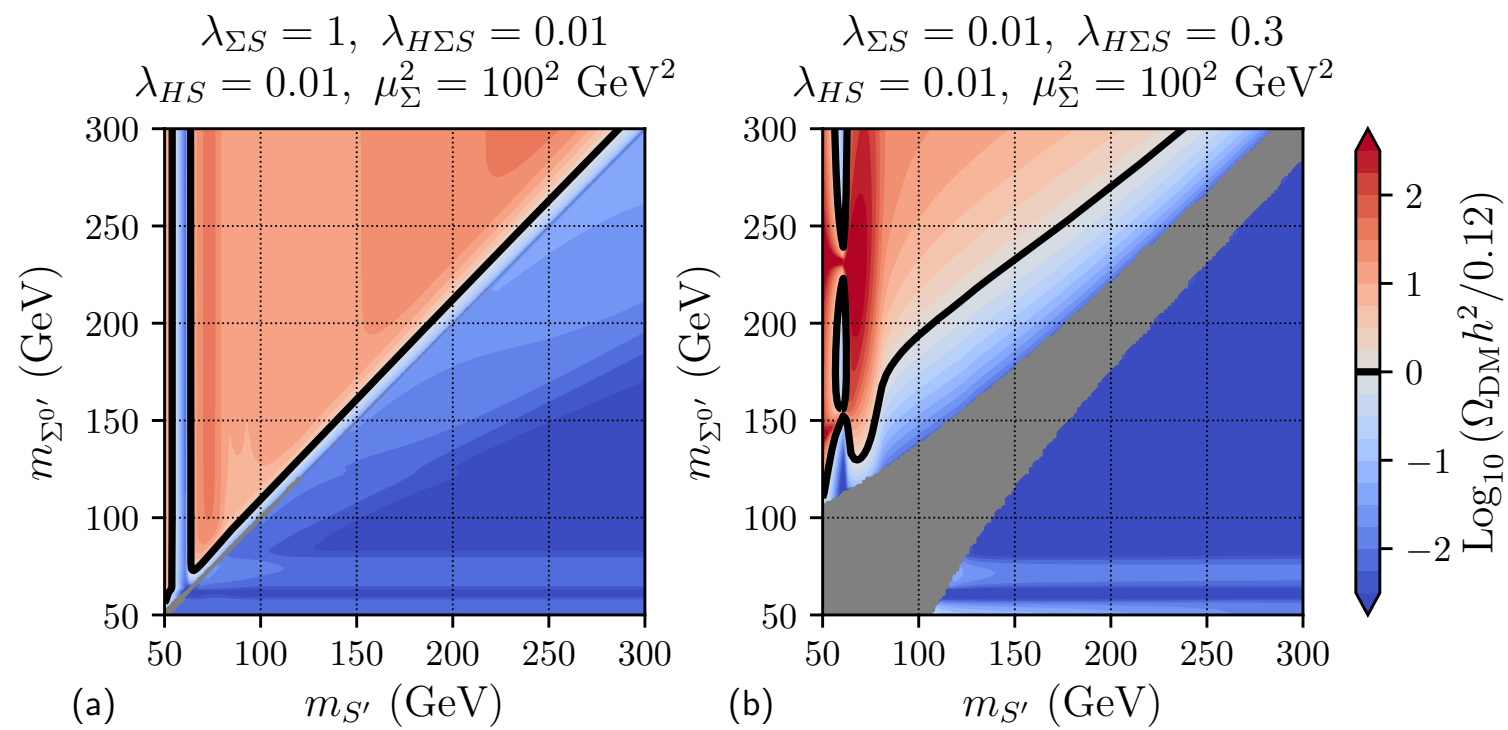

Figure 4. The log of the dark matter relic density normalised to the observed, as a function of the masses of the two $m_{S^{\prime}}$ and $m_{\Sigma^{\prime}}$ for two sets of benchmark scalar couplings. Both $\lambda_{\Sigma}$ and $\lambda_{S}$ have negligible impact on dark matter phenomenology and were set to 0.01 . Red regions indicate a DM over-density, while blue regions indicate an under-density. The solid black line is the relic density contour corresponding to the observed relic density. The grey region along $m_{\Sigma^{0^{\prime}}}=m_{S^{\prime}}$ corresponds to the unphysical region excluded by eq. (2.12).

$m_{S^{\prime}} \lesssim \frac{m_{H}}{2}$. As mentioned previously, this latter region is strongly constrained by invisible Higgs decays and was omitted the from random scan. The benchmark scalar couplings used in figures $4 \mathrm{~b}$ and $5 \mathrm{~b}$ correspond to the second category, where the large neutral scalar large mixing angle allows the correct relic density to be obtained even when $\Delta m$ is large. Once again, the region with $m_{S^{\prime}} \lesssim \frac{m_{H}}{2}$ can yield the correct relic density, though in this case the phenomenology of this region is more complex due to the presence of a large scalar mixing angle. In particular, the $S^{\prime}-H$ quartic coupling, which is given by

$$
\lambda_{H S} \cos ^{2} \theta_{S}-\lambda_{H \Sigma S} \cos \theta_{S} \sin \theta_{S}+\lambda_{H \Sigma} \sin ^{2} \theta_{S},
$$

can be equal to zero for some values of $\theta_{S}$. While this can eliminate the invisible Higgs decay constraint, it also results in an over-density of DM, such that these cancellations can be clearly seen in figure $4 \mathrm{~b}$ near $\left(m_{S^{\prime}}, m_{\Sigma^{0^{\prime}}}\right)=(60 \mathrm{GeV}, 150 \mathrm{GeV})$ and $(60 \mathrm{GeV}, 230 \mathrm{GeV})$. Furthermore, this region of parameter space is nearly excluded by indirect detection constraints. Therefore, we will continue to focus on parameter space with $m_{S^{\prime}}>\frac{m_{H}}{2}$.

We have shown that the $\Sigma x \mathrm{SM}$ is capable of satisfying DM direct detection constraints while generating the observed DM density for a wide range of scalar couplings and masses. However, as discussed in section 1, pure $\mathrm{SU}(2)$ triplet scalar extensions face severe constraints due to collider searches, with a large portion of the triplet masses considered here excluded in minimal triplet extensions. Therefore, in the next section we will examine the collider phenomenology of the $\Sigma x \mathrm{SM}$. 


\section{Direct and Indirect Detection Constraints}
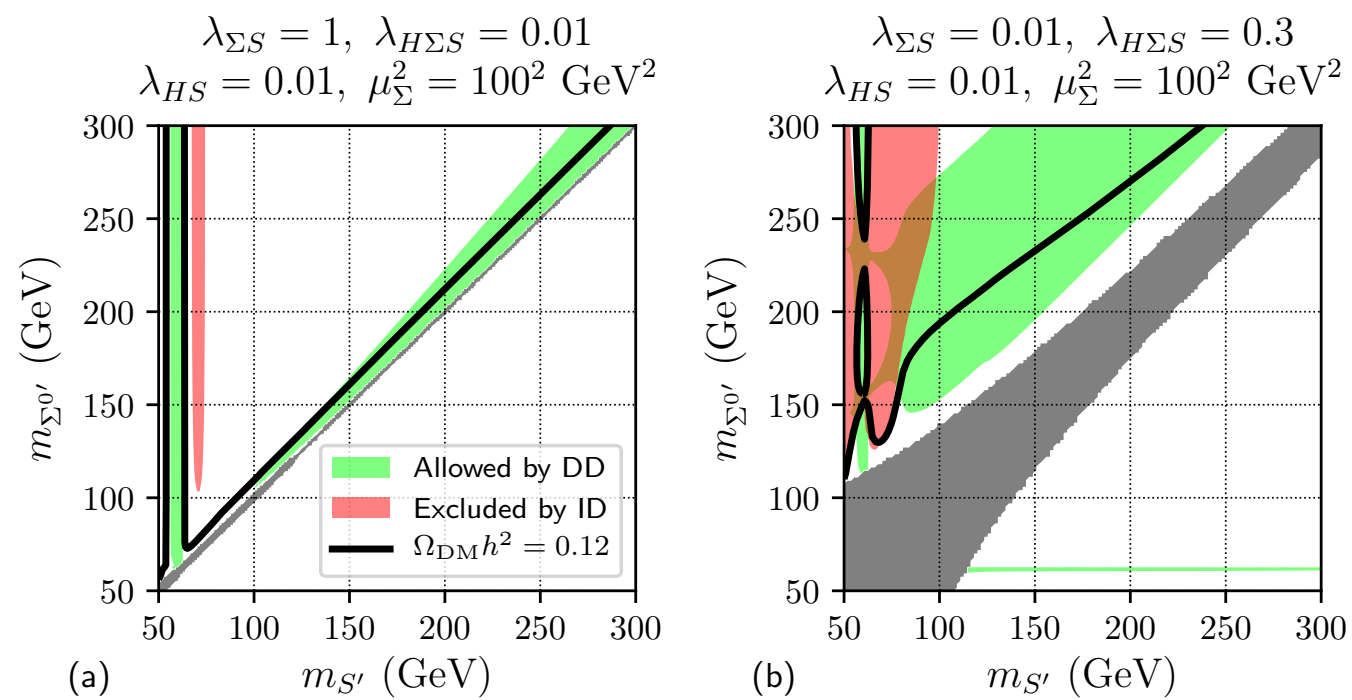

Figure 5. The region of parameter space consistent with the current direct and indirect detection limits as a function of the masses of the two neutral scalars for two sets of benchmark scalar couplings. Both $\lambda_{\Sigma}$ and $\lambda_{S}$ have negligible impact on dark matter phenomenology and were set to 0.01 . The green region is allowed by direct detection constraints, the red region is excluded by indirect detection, and the black line gives the contour that yields the correct DM density. The grey region along $m_{\Sigma^{0^{\prime}}}=m_{S^{\prime}}$ corresponds to the unphysical region excluded by eq. (2.12). Electroweak precision observables are not constraining in these regions of parameter space.

\section{Collider phenomenology}

The main collider signatures of this model can be summarised as follows:

- The new scalars are produced in pairs, primarily through charged and neutral current Drell-Yan processes.

- They decay via their couplings with other scalars and weak gauge bosons, producing lighter scalars and on- or off-shell SM Higgs and weak gauge bosons.

- Due to the $\mathbb{Z}_{2}$ symmetry, the pair production of the new scalar will always result in a final state with at least two stable neutral scalars. ${ }^{3}$

- Therefore, pair production events will always result in large missing energy alongside decay products from the on- or off-shell SM Higgs and weak gauge bosons.

There are several collider searches that are applicable to such signal events. Many of these are supersymmetry searches looking for charginos and neutralinos, with a stable lightest neutralino. This is a consequence of the fact that the production and decay of $\Sigma^{+} \Sigma^{-}, \Sigma^{ \pm} \Sigma^{0^{\prime}}$, and $\Sigma^{ \pm} S^{\prime}$ pairs is analogous to the production and decay of chargino and neutralino pairs, with large missing energy due to a stable neutralino.

\footnotetext{
${ }^{3}$ The $\Sigma^{ \pm}$is never stable due to the radiative mass splitting of the triplet components.
} 


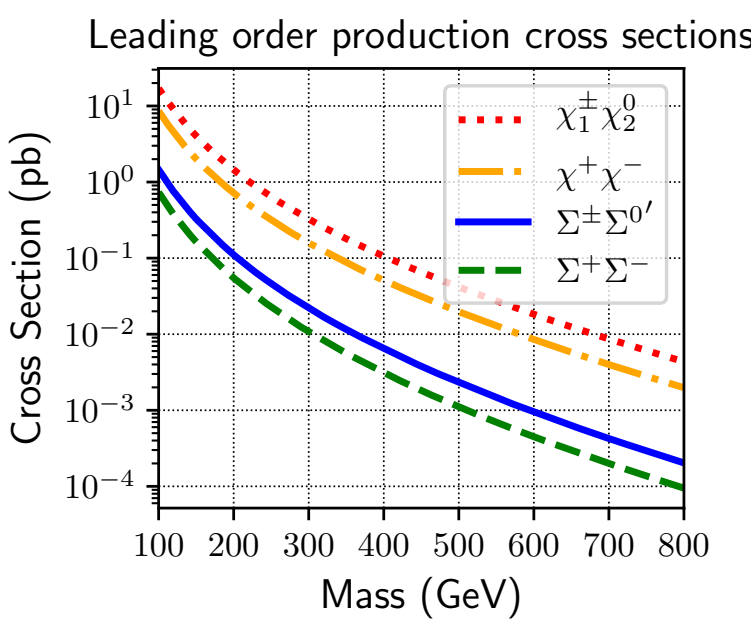

Figure 6. Leading order production cross sections of $\Sigma^{ \pm} \Sigma^{0^{\prime}}$ (solid blue line) and $\Sigma^{+} \Sigma^{-}$(dashed green line) pairs. Note that for this plot the scalar mixing angle was set to zero, such that the $\Sigma^{ \pm} S^{\prime}$ production cross section is zero. For comparison, we also show the $\chi_{1}^{ \pm} \chi_{2}^{0}$ (dotted red line) and $\chi_{1}^{+} \chi_{1}^{-}$(dash-dotted orange line) production cross sections. The $\mathrm{x}$-axis corresponds to the mass of the pair produced particles, with $m_{\chi_{1}^{ \pm}}=m_{\chi_{2}^{0}}$ and $m_{S^{\prime}}=m_{\Sigma^{ \pm}}$. The scalar cross sections were calculated using MadGraph5_aMC@NLO 2.6.5 f [121], and the supersymmetric cross sections obtained using Prospino 2.1 [122].

However, there are some notable differences between the scalars and the chargino/neutralinos, such that the constrains obtained in these SUSY searches are not directly applicable:

- The production cross section of the charginos/neutralinos is larger than our scalar production cross section by a factor of 10-20, resulting in significantly weaker constraints. ${ }^{4}$ The leading order production cross sections are shown in figure 6 ,

- The kinematic distributions are slightly different. The scalars are produced with a harder $p_{t}$ spectrum, which generally leads to more stringent bounds.

- The neutralinos can decay via an (off-shell) $Z$ boson. However, there is no analogous $\Sigma^{0^{\prime}} \rightarrow S^{\prime} Z^{(*)}$ decay. This is due to the fact that the neutral component of a hypercharge-zero triplet does not couple to the $Z$.

The first point, is not an obstacle to the applicability of chargino/neutralino searches, the cross section limits would still apply and would simply lead to less severe limits on the masses. The effect of the second point is difficult to address without using Monte Carlo event generators and re-implementing each of the relevant analyses. The third point is more problematic, as it directly affects the search strategies of the analyses looking for charginos/neutralinos. In order to address the applicability of these chargino/neutralino searches we now discuss the decays of the scalars in more detail.

\footnotetext{
${ }^{4}$ This difference is mainly due to kinematic differences between fermions and scalars. For example, the top quark production cross section is similarly larger than that of an equal mass stop.
} 


\subsection{Decays}

Motivated by the dark matter phenomenology discussed in section 3, we will focus purely on the parameter space where $\Delta m>0$. Therefore, the $S^{\prime}$ is always stable, as it is the lightest particle charged under our $\mathbb{Z}_{2}$ symmetry. The $\Sigma^{+}$can then decay in two ways, via $\Sigma^{+} \rightarrow W^{+(*)} S^{\prime}$ or $\Sigma^{+} \rightarrow W^{+(*)} \Sigma^{0^{\prime}}$. The former decay is suppressed by the neutral scalar mixing angle, while the latter is only kinematically allowed due to the small radiatively induced mass splitting, $m_{\Sigma^{+}}-m_{\Sigma^{0^{\prime}}} \lesssim 166 \mathrm{MeV}$. For most of the parameter space, the kinematic suppression is stronger than the mixing angle suppression, such that $\Sigma^{ \pm}$almost always decays into $W^{+(*)} S^{\prime}$. As a consequence of there only being one decay channel, the branching ratios of the $\Sigma^{+}$decays are not sensitive to the scalar coupling parameters. However, they still determine the $\Sigma^{+}$lifetime. Fortunately the relevant SUSY searches feature analogous chargino decays $\left(\chi_{1}^{+} \rightarrow W^{+(*)} \chi_{1}^{0}\right)$. Therefore, except for some differences in the kinematic distributions due to the fermionic nature of charginos, the upper bound on the production cross section from searches featuring only charginos are directly applicable to $\Sigma^{+}$

The decays of the triplet-like neutral scalar $\Sigma^{0^{\prime}}$ are more complicated. If $\Delta m \gtrsim 10 \mathrm{GeV}$, they will predominantly decay into a $S^{\prime}$ and an (off-shell) SM Higgs. However, for smaller $\Delta m$, they may instead decay via $\Sigma^{0^{\prime}} \rightarrow \Sigma^{ \pm} W^{\mp^{*}}$. Figure 7 shows how the branching ratios and lifetimes of $\Sigma^{0^{\prime}}$ and $\Sigma^{+}$vary as a function of $\Delta m$, for a set of benchmark scalar couplings. The widths were evaluated at leading order using the MadWidth [123] component of MadGraph. Two key features can be seen in figures $7 \mathrm{a}$ and $7 \mathrm{~b}$ :

- There is kinematic suppression of the $\Sigma^{0^{\prime}} \rightarrow S^{\prime} h^{*} \rightarrow S^{\prime} b \bar{b}$ channel for small $\Delta m$.

- The $\Sigma^{0^{\prime}} \rightarrow \Sigma^{ \pm} W^{\mp *}$ partial width and branching ratios are large for small $\Delta m$.

To explain this latter feature, note that this decay can only take place when $m_{\Sigma^{0^{\prime}}}>m_{\Sigma^{ \pm}}$. This mass hierarchy is contrary to what is seen in minimal triplet scalar models, where a radiatively induced mass splitting always leads to $m_{\Sigma^{0^{\prime}}}<m_{\Sigma^{ \pm}}$. As is discussed in section 2.4.1 and illustrated in figure 2, $m_{\Sigma^{0^{\prime}}}>m_{\Sigma^{ \pm}}$only occurs in our model when the neutral scalar mixing raises the mass of the $m_{\Sigma^{0^{\prime}}}$ and overcomes this radiative mass splitting. The effect of the scalar mixing is such that $m_{\Sigma^{0^{\prime}}}-m_{\Sigma^{ \pm}}$is maximised when $|\Delta m|$ is minimised. Therefore, the $\Sigma^{0^{\prime}} \rightarrow \Sigma^{ \pm} W^{\mp^{*}}$ partial width in figure $7 \mathrm{~b}$ is also maximised when $\Delta m$ is minimised. This contrasts with the $\Sigma^{0^{\prime}} \rightarrow S^{\prime} h^{*}$ partial widths, which increase with larger $\Delta m$. These competing effects result in the $\Sigma^{0^{\prime}}$ lifetime featuring a local maximum that can be seen in figure 7c. For this particular set of scalar couplings, the lifetime can be as large as $c \tau \approx 0.2 \mathrm{~mm}$. Given that both of the neutral scalars are stable when $\lambda_{H \Sigma S}=0$, the lifetime can be made arbitrarily large by simply decreasing $\lambda_{H \Sigma S}$.

Once again comparing the decays to supersymmetric searches, we find that when the $\Delta m>m_{H}$, such that $\Sigma^{0^{\prime}}$ decays can produce an on-shell SM Higgs, there exist collider searches that have analogous decays for neutralinos. For example, refs. [124, 125] map neatly onto our model. Unfortunately the searches relevant to small mass differences, refs. [126, 127], have the neutralino decay through an off-shell $Z$, not an off-shell SM Higgs. While searches using the $Z^{*} \rightarrow b \bar{b}$ or $\tau^{+} \tau^{-}$decay processes could easily be applied to our model, the relevant decays instead use $Z^{*} \rightarrow \ell^{+} \ell^{-}$. Therefore interpreting these results will not be as straightforward. 
$\Sigma$ decays with $m_{\Sigma^{ \pm}}=150 \mathrm{GeV}$ and $\lambda_{H \Sigma S}=0.05$
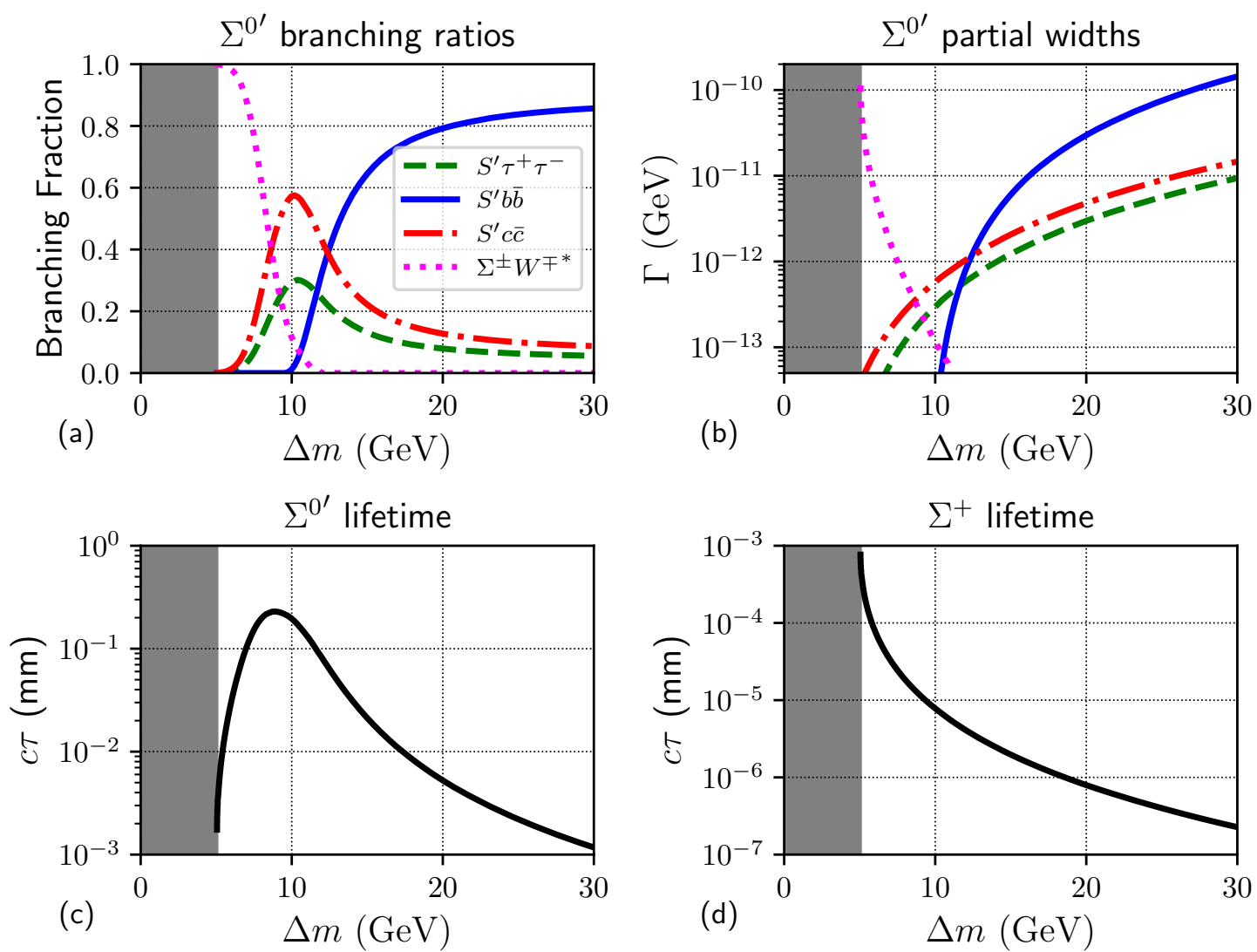

Figure 7. Plots show the branching ratios (a) and partial widths (b) of $\Sigma^{0^{\prime}}$ decays, along with the lifetimes of the $\Sigma^{0^{\prime}}$ (c) and $\Sigma^{+}(\mathrm{d})$. The branching ratios of the $\Sigma^{+}$decays are not shown as they do not change significantly as a function of $\Delta m$. The solid grey area indicates the unphysical region excluded by eq. (2.12). All scalar couplings were held constant, and $\Delta m$ was varied by changing only $\mu_{S}^{2}$.

\subsection{Collider searches}

Obtaining precise collider limits requires generating events for a given model, applying the analyses performed in relevant collider searches, and comparing the predicted number of events with those observed by the relevant searches. Fortunately, there exist useful tools, such as CheckMATE [128], that implement existing collider searches and make it straightforward to recast their results onto new models. Unfortunately, the searches most relevant to our model, particularly refs. [124-127, 129], have not yet been implemented in these tools. ${ }^{5}$ Implementation of all of these analyses requires a considerable amount of work, with some analyses featuring up to 58 signal regions. For the sake of this initial investigation, we will limit the scope of our collider phenomenology to re-scaling the bounds on charginos and neutralinos obtained by these analyses and leave a more thorough investigation as potential future work.

\footnotetext{
${ }^{5}$ Some older and superseded searches are implemented by these tools, but in a preliminary scan they were not able exclude any parameter space with $m_{\Sigma^{0^{\prime}}}, m_{\Sigma^{+}}>100 \mathrm{GeV}$.
} 
As discussed in the previous section, the dark matter direct detection constraints, together with the requirement that our dark matter candidate constitutes the whole relic density, imply that either the neutral scalar mass difference is small, $0<\Delta m \lesssim 40 \mathrm{GeV}$, or there is a large Higgs coupling $\lambda_{H \Sigma S} \gtrsim 0.5$ and mixing angle $\left|\sin \theta_{S}\right| \gtrsim 0.3$. The two parameter space possibilities are constrained by different collider searches and will be discussed separately with reference to relevant chargino and neutralino searches.

\subsubsection{Small mass difference, $\Delta m<50 \mathrm{GeV}$}

Searching for charginos and neutralinos with compressed mass spectra, mass difference less than $\sim 50 \mathrm{GeV}$, is notoriously difficult. The main challenge boils down to the fact that the visible decay products have low energies due to the limited energy budget, even when boosted by initial-state radiation jets. Both the CMS [127] and ATLAS [126] collaborations have undertaken searches for this type of supersymmetric spectrum, using $36 \mathrm{fb}^{-1}$ and $140 \mathrm{fb}^{-1}$ of data respectively, with the more recent ATLAS search setting more stringent bounds.

The problem of low-energy visible particles also holds for our scalars. However, we have the added complication that existing searches use the $\chi_{2}^{0} \rightarrow \chi_{1}^{0} Z^{*} \rightarrow \chi_{1}^{0} \ell^{+} \ell^{-}$decay channel. Our best analogue is the $\Sigma^{0^{\prime}} \rightarrow S^{\prime} h^{*} \rightarrow S^{\prime} \tau^{+} \tau^{-}$channel with both of the taus decaying leptonically into a same-flavor opposite-charge light lepton pair. The total branching ratio for this process can be as large as $\sim 2 \%{ }^{6}$ This is smaller than the branching ratio for off-shell $Z$ decays into electrons or muons, which ranges from $7 \%$ to $10 \%$ [126].

Additionally, relying on the leptonic tau decays to produce light leptons further reduces the energy of the light leptons, due to some of the $\Sigma^{0^{\prime}}$ decay energy budget going into the neutrinos produced in the tau decay. Thus, ignoring all other factors, we would expect weaker limits on the production cross section. This is slightly offset by the fact that the scalar $p_{T}$ spectrum has a slightly larger high energy tail than the charginos and neutralinos do. However given that most of this energy is taken away by the $S^{\prime}$ it is likely that the net result is that the model is more difficult to probe.

Figure 8 shows the distribution of the $\tau$ pair and light lepton-pair invariant masses in the scalar model, obtained using MadGraph, Pythia [130], and MadAnalysis [131]. This figure is analogous to figure 2 in ref. [126]. Note that our $m_{\tau \tau}$ closely corresponds to the $m_{\ell \ell}$ spectrum computed in ref. [126] for the wino-bino scenario with $m_{\chi_{2}^{0}} \times m_{\chi_{1}^{0}}>0$. However, our actual $m_{\ell \ell}$ spectrum, where the light leptons arise from leptonic tau decays, is skewed towards smaller values due to energy taken away by the neutrinos. The effect for this analysis may be that scalars with a given mass difference should be compared to charginos and neutralinos with some smaller mass difference due to the higher proportion of low $m_{\ell \ell}$ events. While this may require us to modify the production cross section limits as a function of $\Delta m$, this should not result in a larger maximal mass reach.

As is discussed in section 4.1, the $\Sigma^{0^{\prime}} \rightarrow \Sigma^{ \pm} W^{\mp^{*}} \rightarrow S^{\prime} W^{ \pm *} W^{\mp^{*}}$ decay channel branching fraction can be large when $\Delta m \lesssim 10 \mathrm{GeV}$. This decay can also produce two oppositesign same-flavour leptons and contribute signal events. However, as the branching ratio for

\footnotetext{
${ }^{6}$ This branching ratio is maximised when the masses and $\lambda_{H \Sigma S}$ are selected such that $\operatorname{Br}\left(\Sigma^{0^{\prime}} \rightarrow \Sigma^{ \pm} W^{\mp^{*}}\right)=0$ and $\operatorname{Br}\left(\Sigma^{0^{\prime}} \rightarrow S^{\prime} \tau^{+} \tau^{-}\right) \sim 30 \%$.
} 


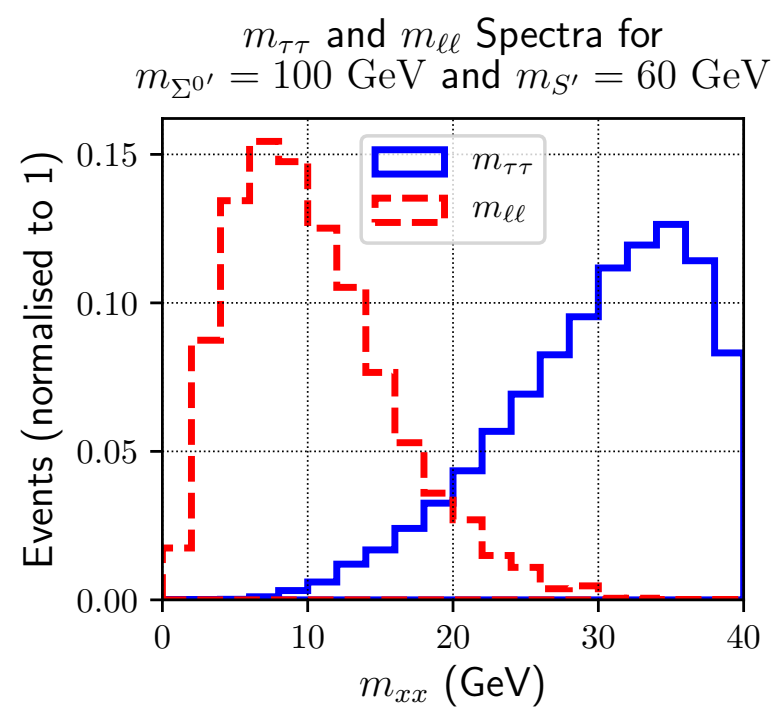

Figure 8. Normalised spectrum of tau pair (solid blue line) and light lepton pair (dashed red line) invariant masses, where the light leptons are the products of leptonic tau decays. This figure is analogous to figure 2 in ref. [126], however our $m_{\ell \ell}$ spectrum is skewed towards smaller energies.

this decay increases, the $\Sigma^{0^{\prime}} \rightarrow S^{\prime} \tau^{+} \tau^{-}$branching ratio decreases. The result is that the total branching ratio to produce opposite-sign same-flavour lepton pairs remains below $7 \%$. Furthermore, this channel faces the same challenge of losing some of its limited energy budget to neutrinos. This is compounded by the fact that an appreciable branching fraction necessarily requires $\Delta m$ to be very small. The resulting visible lepton energy spectra are thus shifted towards energies in a manner similar to those arising from leptonic tau decays.

If we ignore the differences in the visible particle $p_{T}$ spectra, then in principle one could use the $95 \%$ confidence upper bounds on chargino production reported in ref. [126] to approximate the constraints on our new scalars. Given that our branching fraction to produce the necessary lepton pairs is always smaller than for charginos and neutralinos, we can get conservative constraints by simply using our scalar production cross sections as shown in figure $6 .{ }^{7}$ We find that none of the upper limits on the chargino-neutralino production cross section that are provided by the ATLAS analysis $[132,133]$ are capable of excluding the scalars. Given that we do not expect the visible particle $p_{T}$ spectrum differences to increase the mass reach of this analysis, we are confident in saying that the compressed mass spectrum searches do not currently constrain this model when $m_{\Sigma^{0^{\prime}}}>100 \mathrm{GeV}$.

\subsubsection{Large mass difference, $\Delta m>m_{H}$}

When the neutralino mass difference is large, an on-shell SM Higgs boson can be produced in the decay of neutralinos. Both the CMS [124] and ATLAS [125] collaborations have searches looking for this type of decay using $36 \mathrm{fb}^{-1}$ of data. There are also some more

\footnotetext{
${ }^{7}$ Note that whenever the scalar mixing angle is large, the production cross section will be even smaller.
} 
recent searches looking for diphoton signals in $\chi_{2}^{0} \rightarrow \chi_{1}^{0} h \rightarrow \chi_{1}^{0} \gamma \gamma$, with a $77.5 \mathrm{fb}^{-1} \mathrm{CMS}$ search [129] and a $140 \mathrm{fb}^{-1}$ ATLAS search [134].

Fortunately, unlike the compressed mass spectra searches, these types of searches are more directly analogous to the decay processes that are present in our scalar model. Repeating what was done in the previous section and simply directly using the $\Sigma^{ \pm} \Sigma^{0^{\prime}}$ production cross section shown in figure 6 , we find that none of the upper-limits provided by these searches exclude our Type-IV $\Sigma x$ SM. The exclusion boundary lies below the mass ranges considered by ATLAS and CMS. Furthermore, the production cross section in figure 6 is for $\theta_{S}=0$, which maximises the number of signal events. Given that DM constraints require $\mid \sin \theta_{S} \gtrsim 0.3$ for $\Delta m \gtrsim 40 \mathrm{GeV}$, the actual production cross section for signal events is even smaller.

The more recent $\chi_{2}^{0} \rightarrow \chi_{1}^{0} h \rightarrow \chi_{1}^{0} \gamma \gamma$ searches, refs. [129, 134], are similarly insensitive. They are only able to exclude neutralinos with $\chi_{1}^{0} \lesssim 100 \mathrm{GeV}$, and have no sensitivity to scalars with $m_{S^{\prime}} \sim 100-300 \mathrm{GeV}$. It should be noted that diphoton signal regions were included in the $36 \mathrm{fb}^{-1}$ ATLAS and CMS searches. However, compared to signal regions with $H \rightarrow b \bar{b}$, they were relatively insensitive. Upcoming $H \rightarrow b \bar{b}$ searches with the full Run 2 data set may place stronger constraints on our scalars.

\subsubsection{Intermediate mass difference, $50 \mathrm{GeV}<\Delta m<m_{H}$}

When $\Delta m>50 \mathrm{GeV}$, compressed mass spectra searches become insensitive due to cuts that are designed for low-energy visible particles. However, the large mass-difference searches with an on-shell SM Higgs only apply when $\Delta m>m_{H}$. For weak-scale mass differences, the chargino and neutralino searches generally focus on $\chi_{2}^{0} \rightarrow Z^{(*)} \chi_{1}^{0}$ [135]. If the neutralino mass difference is larger than $m_{Z}$, then these searches generally involve reconstructing an on-shell $Z$. Therefore, these searches are not applicable to our model due to the lack of a $\Sigma^{0^{\prime}} \rightarrow Z S^{\prime}$ decay. If the $Z$ is off-shell, we can use the same approach as in section 4.2.1. That is, we could once again use the $\Sigma^{0^{\prime}} \rightarrow S^{\prime} h^{*} \rightarrow S^{\prime} \tau \tau$ decay to the generate a reduced number signal events, but the result will be similarly unconstraining.

One type of analysis we have not yet discussed is a search for chargino pair production, which looks for $p p \rightarrow \chi_{1}^{+} \chi_{1}^{-} \rightarrow \chi_{1}^{0} \chi_{1}^{0} W^{+} W^{-}$. This is directly analogous to $\Sigma^{+} \Sigma^{-}$pair production, and therefore such searches could conceivably constrain the scalars when $\Delta m>$ $m_{W}$. In particular, there is a recent ATLAS search looking for this type of signal using $140 \mathrm{fb}^{-1}$ of data [136], which provides upper limits on the chargino pair production cross section [137]. However, once again, interpretation of this data in terms of the $\Sigma^{+} \Sigma^{-}$pair production cross section results in no limits on the charged triplets.

\subsection{Alternative searches and future prospects}

In addition to the SUSY searches outlined above, there are several other methods that could be employed to search for the $\Sigma x \mathrm{SM}$. One such method would be to search for displaced vertices. Displaced vertices are the result of a long lived particle that travels a significant distance from the main interaction vertex before decaying. As mentioned in section 4.1, the lifetime of the $\Sigma^{0^{\prime}}$ can be made arbitrarily large by making $\lambda_{H \Sigma S}$ arbitrarily small. Thus, in principle, there are regions of parameter space that would generate displaced vertices. 
However, the low energy of the visible decay products, which is due to the small $\Delta m$, poses a major challenge. Existing displaced vertex searches generally place minimum $p_{T}$ cuts that would eliminate any signal events generated by a long lived $\Sigma^{0^{\prime}}$.

Another method of improving the discoverability of the $\Sigma x \mathrm{SM}$ would be to eliminate the sub-optimal reliance on chargino and neutralino based searches. In particular, for the compressed mass spectrum scenario, the fact that existing searches generally look for light lepton pairs coming from an off-shell $Z^{*}$ decay, means they are ill suited to our model, which relies on $h^{*}$ decay products. More suitable searches may instead target pairs of low energy $b$ jets, hadronically decaying taus, or taus decaying leptonically into different lepton flavours. The $\Sigma^{0^{\prime}} \rightarrow \Sigma^{ \pm} W^{\mp^{*}}$ decay, which also has no neutralino analogue, may also allow for alternative searches using $\Sigma^{ \pm} \Sigma^{0^{\prime}} \rightarrow S^{\prime} S^{\prime} W^{ \pm *} W^{ \pm *} W^{\mp^{*}}$ events. However, as was mentioned before, this decay channel is only present when the mass splitting is very small. Also note that a more thorough analysis that relies on this branching ratio at small mass splittings likely requires a more precise treatment of the radiative mass splitting than the approximation we made in section 2.4.1. This reliance on chargino and neutralino oriented searches also applies to the intermediate mass difference scenario.

However, even with searches optimised to look for this particular model, the constraints on these scalars will never be quite as strong as those that apply to charginos and neutralinos. This is simply due to the fact that the chargino and neutralino production cross section are roughly a factor of 10-20 larger than the triplet-scalar production cross section. Therefore, constraints on the scalars with $140 \mathrm{fb}^{-1}$ of data available are comparable to those that would be obtained in chargino and neutralino searches using $\lesssim 14 \mathrm{fb}^{-1}$ of data. Given that current chargino and neutralino searches are only just starting to probe neutralino masses around 100-200 GeV, the parameter space considered when discussing the dark matter phenomenology in section 3 will likely only begin to be thoroughly probed by analyses using at least $1000 \mathrm{fb}^{-1}$ of data.

\section{Conclusion}

We have shown that, by introducing a scalar singlet, one is able to relax the collider constraints on minimal SU(2) triplet scalar extensions of the SM. Furthermore, such a model is capable of generating the correct relic density. We have only examined one type of such a model, where both the triplet and singlet scalars are charged under a single $\mathbb{Z}_{2}$ symmetry. There exist several other variants depending on how $\mathbb{Z}_{2}$ charges are assigned. Examination of these models, or a more robust examination of the collider phenomenology of this model are potential avenues of further investigation.

This paper focused solely on the collider and dark matter phenomenology, but was motivated by the prospect of novel electroweak phase transitions. The relaxation of collider constraints, combined with the new interactions with the scalar singlet, opens greater parameter space for such transitions. However, if one requires these new scalars to constitute all of the dark matter density we must impose new constraints, namely that either $m_{\Sigma^{0^{\prime}}}-m_{S^{\prime}} \sim 10$ to $30 \mathrm{GeV}$, or $\lambda_{H \Sigma S} \gtrsim 0.5$. The natural next step is a detailed examination of the phase transition dynamics to establish if a novel phase transition can indeed be obtained in some region of this enlarged parameter space. 


\section{A Loop induced annihilation into monochromatic photons}

Our dark matter can annihilate into two photons via an off-shell intermediate Higgs boson. This annihilation process has been studied previously in both minimal triplet [96] and minimal singlet $[138,139]$ scalar DM models. The cross section for annihilation into monochromatic photons is given by [96, 138, 139],

$$
\sigma\left(X X \rightarrow h^{*} \rightarrow \gamma \gamma\right)=\frac{\lambda_{H X}^{2}}{\sqrt{s-4 m_{X}^{2}}} \frac{\Gamma_{h^{*} \rightarrow \gamma \gamma}(s)}{\left(s-m_{H}^{2}\right)^{2}+m_{H}^{2} \Gamma_{h}^{2}},
$$

where $X$ is either $\Sigma^{0^{\prime}}$ or $S^{\prime}$, and

$$
\begin{gathered}
\lambda_{H S^{\prime}}=\lambda_{H S} \cos ^{2}\left(\theta_{S}\right)-2 \lambda_{H \Sigma S} \cos \left(\theta_{S}\right) \sin \left(\theta_{S}\right)+\lambda_{H \Sigma} \sin ^{2}\left(\theta_{S}\right), \\
\lambda_{H \Sigma^{0^{\prime}}}=\lambda_{H \Sigma} \cos ^{2}\left(\theta_{S}\right)+2 \lambda_{H \Sigma S} \cos \left(\theta_{S}\right) \sin \left(\theta_{S}\right)+\lambda_{H S} \sin ^{2}\left(\theta_{S}\right) .
\end{gathered}
$$

The off-shell Higgs diphoton rate is given by,

$$
\begin{aligned}
\Gamma_{h^{*} \rightarrow \gamma \gamma}(s)=\frac{\alpha^{2} s^{3 / 2}}{256 \pi^{3} v_{H}^{2}} & \mid \frac{4}{3} A_{1 / 2}\left(\frac{s}{4 m_{t}}\right)+A_{1}\left(\frac{s}{4 m_{w}}\right) \\
& +\left.\frac{2 v_{H} \lambda_{H \Sigma m_{W}}}{g m_{\Sigma^{+}}^{2}} A_{0}\left(\frac{s}{4 m_{\Sigma^{+}}}\right)\right|^{2},
\end{aligned}
$$

with the loop functions,

$$
\begin{aligned}
A_{0}(x) & =-(x-f(x)) x^{-2}, \\
A_{1 / 2}(x) & =2(x+(x-1) f(x)) x^{-2}, \\
A_{1}(x) & =-\left(3 x+2 x^{2}+3(2 x-1) f(x)\right) x^{-2}, \\
f(x) & =\left\{\begin{array}{ll}
\arcsin ^{2}(\sqrt{x}) & x \leq 1 \\
-\frac{1}{4}\left(\ln \frac{1+\sqrt{1-x^{-1}}}{1-\sqrt{1-x^{-1}}}-i \pi\right)^{2} & x>1
\end{array} .\right.
\end{aligned}
$$

If we set $\theta_{S}=0$ and make the triplet very heavy, our diphoton annihilation rates are consistent with the minimal singlet scalar DM annihilation rates in refs. [138] and [139].

In order to get our Fermi-LAT constraint we then evaluate $\sigma v$ in the zero-velocity limit $\left(s \rightarrow 4 m_{X}^{2}\right)$. If our model results in a DM under-density, such that it only contributes a fraction of the DM density, we scale the annihilation rate by $\left(\frac{\Omega_{\mathrm{DM}} h^{2}}{0.12}\right)^{2}$. 
Open Access. This article is distributed under the terms of the Creative Commons Attribution License (CC-BY 4.0), which permits any use, distribution and reproduction in any medium, provided the original author(s) and source are credited.

\section{References}

[1] M. Trodden, Electroweak baryogenesis: A Brief review, in 33rd Rencontres de Moriond: Electroweak Interactions and Unified Theories (1998) [hep-ph/9805252] [INSPIRE].

[2] D.E. Morrissey and M.J. Ramsey-Musolf, Electroweak baryogenesis, New J. Phys. 14 (2012) 125003 [arXiv: 1206.2942 ] [INSPIRE].

[3] D.J. Weir, Gravitational waves from a first order electroweak phase transition: a brief review, Phil. Trans. Roy. Soc. Lond. A 376 (2018) 20170126 [arXiv:1705. 01783] [InSPIRE].

[4] A. Mazumdar and G. White, Review of cosmic phase transitions: their significance and experimental signatures, Rept. Prog. Phys. 82 (2019) 076901 [arXiv: 1811. 01948] [INSPIRE].

[5] M.J. Ramsey-Musolf, The electroweak phase transition: a collider target, JHEP 09 (2020) 179 [arXiv: 1912.07189] [INSPIRE].

[6] K. Kajantie, M. Laine, K. Rummukainen and M.E. Shaposhnikov, A Nonperturbative analysis of the finite $T$ phase transition in $\mathrm{SU}(2) \times \mathrm{U}(1)$ electroweak theory, Nucl. Phys. $B$ 493 (1997) 413 [hep-lat/9612006] [INSPIRE].

[7] K. Kajantie, M. Laine, K. Rummukainen and M.E. Shaposhnikov, Is there a hot electroweak phase transition at $m_{H} \gtrsim m_{W}$ ?, Phys. Rev. Lett. 77 (1996) 2887 [hep-ph/9605288] [INSPIRE].

[8] M. Gurtler, E.-M. Ilgenfritz and A. Schiller, Where the electroweak phase transition ends, Phys. Rev. D 56 (1997) 3888 [hep-lat/9704013] [INSPIRE].

[9] M. Laine and K. Rummukainen, What's new with the electroweak phase transition?, Nucl. Phys. B Proc. Suppl. 73 (1999) 180 [hep-lat/9809045] [inSPIRE].

[10] F. Csikor, Z. Fodor and J. Heitger, Endpoint of the hot electroweak phase transition, Phys. Rev. Lett. 82 (1999) 21 [hep-ph/9809291] [INSPIRE].

[11] Y. Aoki, F. Csikor, Z. Fodor and A. Ukawa, The Endpoint of the first order phase transition of the $\mathrm{SU}(2)$ gauge Higgs model on a four-dimensional isotropic lattice, Phys. Rev. D 60 (1999) 013001 [hep-lat/9901021] [INSPIRE].

[12] J.R. Espinosa and M. Quirós, The Electroweak phase transition with a singlet, Phys. Lett. B 305 (1993) 98 [hep-ph/9301285] [INSPIRE].

[13] K.E.C. Benson, Avoiding baryon washout in the extended Standard Model, Phys. Rev. D 48 (1993) 2456 [INSPIRE].

[14] J. Choi and R.R. Volkas, Real Higgs singlet and the electroweak phase transition in the Standard Model, Phys. Lett. B 317 (1993) 385 [hep-ph/9308234] [InSPIRE].

[15] L. Vergara, Baryon asymmetry persistence in the standard model with a singlet, Phys. Rev. D 55 (1997) 5248 [INSPIRE].

[16] S.W. Ham, Y.S. Jeong and S.K. Oh, Electroweak phase transition in an extension of the standard model with a real Higgs singlet, J. Phys. G 31 (2005) 857 [hep-ph/0411352] [INSPIRE].

[17] A. Ahriche, What is the criterion for a strong first order electroweak phase transition in singlet models?, Phys. Rev. D 75 (2007) 083522 [hep-ph/0701192] [INSPIRE]. 
[18] S. Profumo, M.J. Ramsey-Musolf and G. Shaughnessy, Singlet Higgs phenomenology and the electroweak phase transition, JHEP 08 (2007) 010 [arXiv:0705.2425] [INSPIRE].

[19] A. Noble and M. Perelstein, Higgs self-coupling as a probe of electroweak phase transition, Phys. Rev. D 78 (2008) 063518 [arXiv:0711.3018] [INSPIRE].

[20] J.R. Espinosa and M. Quirós, Novel Effects in Electroweak Breaking from a Hidden Sector, Phys. Rev. D 76 (2007) 076004 [hep-ph/0701145] [INSPIRE].

[21] J.R. Espinosa, T. Konstandin, J.M. No and M. Quirós, Some Cosmological Implications of Hidden Sectors, Phys. Rev. D 78 (2008) 123528 [arXiv:0809.3215] [INSPIRE].

[22] V. Barger, P. Langacker, M. McCaskey, M.J. Ramsey-Musolf and G. Shaughnessy, LHC Phenomenology of an Extended Standard Model with a Real Scalar Singlet, Phys. Rev. D 77 (2008) 035005 [arXiv:0706.4311] [INSPIRE].

[23] A. Ashoorioon and T. Konstandin, Strong electroweak phase transitions without collider traces, JHEP 07 (2009) 086 [arXiv:0904.0353] [inSPIRE].

[24] S. Das, P.J. Fox, A. Kumar and N. Weiner, The Dark Side of the Electroweak Phase Transition, JHEP 11 (2010) 108 [arXiv:0910.1262] [INSPIRE].

[25] J.R. Espinosa, T. Konstandin and F. Riva, Strong Electroweak Phase Transitions in the Standard Model with a Singlet, Nucl. Phys. B 854 (2012) 592 [arXiv:1107.5441] [INSPIRE].

[26] J.M. Cline and K. Kainulainen, Electroweak baryogenesis and dark matter from a singlet Higgs, JCAP 01 (2013) 012 [arXiv:1210.4196] [INSPIRE].

[27] D.J.H. Chung, A.J. Long and L.-T. Wang, $125 \mathrm{GeV}$ Higgs boson and electroweak phase transition model classes, Phys. Rev. D 87 (2013) 023509 [arXiv:1209.1819] [INSPIRE].

[28] V. Barger, D.J.H. Chung, A.J. Long and L.-T. Wang, Strongly First Order Phase Transitions Near an Enhanced Discrete Symmetry Point, Phys. Lett. B 710 (2012) 1 [arXiv:1112.5460] [INSPIRE].

[29] W. Huang, J. Shu and Y. Zhang, On the Higgs Fit and Electroweak Phase Transition, JHEP 03 (2013) 164 [arXiv:1210.0906] [InSPIRE].

[30] P.H. Damgaard, D. O'Connell, T.C. Petersen and A. Tranberg, Constraints on New Physics from Baryogenesis and Large Hadron Collider Data, Phys. Rev. Lett. 111 (2013) 221804 [arXiv: 1305.4362] [INSPIRE].

[31] M. Fairbairn and R. Hogan, Singlet Fermionic Dark Matter and the Electroweak Phase Transition, JHEP 09 (2013) 022 [arXiv: 1305.3452] [INSPIRE].

[32] J.M. No and M. Ramsey-Musolf, Probing the Higgs Portal at the LHC Through Resonant di-Higgs Production, Phys. Rev. D 89 (2014) 095031 [arXiv:1310.6035] [INSPIRE].

[33] S. Profumo, M.J. Ramsey-Musolf, C.L. Wainwright and P. Winslow, Singlet-catalyzed electroweak phase transitions and precision Higgs boson studies, Phys. Rev. D 91 (2015) 035018 [arXiv: 1407.5342] [INSPIRE].

[34] N. Craig, H.K. Lou, M. McCullough and A. Thalapillil, The Higgs Portal Above Threshold, JHEP 02 (2016) 127 [arXiv:1412.0258] [InSPIRE].

[35] D. Curtin, P. Meade and C.-T. Yu, Testing Electroweak Baryogenesis with Future Colliders, JHEP 11 (2014) 127 [arXiv:1409.0005] [InSPIRE].

[36] C.-Y. Chen, S. Dawson and I.M. Lewis, Exploring resonant di-Higgs boson production in the Higgs singlet model, Phys. Rev. D 91 (2015) 035015 [arXiv:1410.5488] [INSPIRE]. 
[37] A. Katz and M. Perelstein, Higgs Couplings and Electroweak Phase Transition, JHEP 07 (2014) 108 [arXiv: 1401.1827] [INSPIRE].

[38] J. Kozaczuk, Bubble Expansion and the Viability of Singlet-Driven Electroweak Baryogenesis, JHEP 10 (2015) 135 [arXiv:1506.04741] [INSPIRE].

[39] S. Kanemura, M. Kikuchi and K. Yagyu, Radiative corrections to the Higgs boson couplings in the model with an additional real singlet scalar field, Nucl. Phys. B 907 (2016) 286 [arXiv:1511.06211] [INSPIRE].

[40] P.H. Damgaard, A. Haarr, D. O'Connell and A. Tranberg, Effective Field Theory and Electroweak Baryogenesis in the Singlet-Extended Standard Model, JHEP 02 (2016) 107 [arXiv: 1512.01963] [INSPIRE].

[41] P. Huang, A. Joglekar, B. Li and C.E.M. Wagner, Probing the Electroweak Phase Transition at the LHC, Phys. Rev. D 93 (2016) 055049 [arXiv: 1512.00068] [InSPIRE].

[42] S. Kanemura, M. Kikuchi and K. Yagyu, One-loop corrections to the Higgs self-couplings in the singlet extension, Nucl. Phys. B 917 (2017) 154 [arXiv:1608.01582] [INSPIRE].

[43] A.V. Kotwal, M.J. Ramsey-Musolf, J.M. No and P. Winslow, Singlet-catalyzed electroweak phase transitions in the $100 \mathrm{TeV}$ frontier, Phys. Rev. D 94 (2016) 035022 [arXiv: 1605.06123] [INSPIRE].

[44] T. Brauner, T.V.I. Tenkanen, A. Tranberg, A. Vuorinen and D.J. Weir, Dimensional reduction of the Standard Model coupled to a new singlet scalar field, JHEP 03 (2017) 007 [arXiv: 1609.06230] [INSPIRE].

[45] T. Huang, J.M. No, L. Pernié, M. Ramsey-Musolf, A. Safonov, M. Spannowsky et al., Resonant di-Higgs boson production in the $b \bar{b} W W$ channel: Probing the electroweak phase transition at the LHC, Phys. Rev. D 96 (2017) 035007 [arXiv:1701.04442] [InSPIRE].

[46] C.-Y. Chen, J. Kozaczuk and I.M. Lewis, Non-resonant Collider Signatures of a Singlet-Driven Electroweak Phase Transition, JHEP 08 (2017) 096 [arXiv:1704.05844] [INSPIRE].

[47] A. Beniwal, M. Lewicki, J.D. Wells, M. White and A.G. Williams, Gravitational wave, collider and dark matter signals from a scalar singlet electroweak baryogenesis, JHEP 08 (2017) 108 [arXiv: 1702.06124] [inSPIRE].

[48] J.M. Cline, K. Kainulainen and D. Tucker-Smith, Electroweak baryogenesis from a dark sector, Phys. Rev. D 95 (2017) 115006 [arXiv:1702.08909] [INSPIRE].

[49] G. Kurup and M. Perelstein, Dynamics of Electroweak Phase Transition In Singlet-Scalar Extension of the Standard Model, Phys. Rev. D 96 (2017) 015036 [arXiv:1704.03381] [INSPIRE].

[50] A. Alves, T. Ghosh, H.-K. Guo, K. Sinha and D. Vagie, Collider and Gravitational Wave Complementarity in Exploring the Singlet Extension of the Standard Model, JHEP 04 (2019) 052 [arXiv: 1812.09333] [INSPIRE].

[51] H.-L. Li, M. Ramsey-Musolf and S. Willocq, Probing a scalar singlet-catalyzed electroweak phase transition with resonant di-Higgs boson production in the $4 b$ channel, Phys. Rev. D 100 (2019) 075035 [arXiv: 1906. 05289] [INSPIRE].

[52] O. Gould, J. Kozaczuk, L. Niemi, M.J. Ramsey-Musolf, T.V.I. Tenkanen and D.J. Weir, Nonperturbative analysis of the gravitational waves from a first-order electroweak phase transition, Phys. Rev. D 100 (2019) 115024 [arXiv:1903.11604] [INSPIRE]. 
[53] J. Kozaczuk, M.J. Ramsey-Musolf and J. Shelton, Exotic Higgs boson decays and the electroweak phase transition, Phys. Rev. D 101 (2020) 115035 [arXiv:1911.10210] [INSPIRE].

[54] M. Carena, Z. Liu and Y. Wang, Electroweak phase transition with spontaneous $Z_{2}$-breaking, JHEP 08 (2020) 107 [arXiv:1911.10206] [INSPIRE].

[55] B. Heinemann and Y. Nir, The Higgs program and open questions in particle physics and cosmology, Usp. Fiz. Nauk 189 (2019) 985 [arXiv:1905.00382] [INSPIRE].

[56] N. Turok and J. Zadrozny, Phase transitions in the two doublet model, Nucl. Phys. B 369 (1992) 729 [INSPIRE].

[57] A.T. Davies, C.D. froggatt, G. Jenkins and R.G. Moorhouse, Baryogenesis constraints on two Higgs doublet models, Phys. Lett. B 336 (1994) 464 [InSPIRE].

[58] A. Hammerschmitt, J. Kripfganz and M.G. Schmidt, Baryon asymmetry from a two stage electroweak phase transition?, Z. Phys. C 64 (1994) 105 [hep-ph/9404272] [InSPIRE].

[59] J.M. Cline and P.-A. Lemieux, Electroweak phase transition in two Higgs doublet models, Phys. Rev. D 55 (1997) 3873 [hep-ph/9609240] [INSPIRE].

[60] L. Fromme, S.J. Huber and M. Seniuch, Baryogenesis in the two-Higgs doublet model, JHEP 11 (2006) 038 [hep-ph/0605242] [INSPIRE].

[61] J.M. Cline, K. Kainulainen and M. Trott, Electroweak Baryogenesis in Two Higgs Doublet Models and B meson anomalies, JHEP 11 (2011) 089 [arXiv:1107.3559] [INSPIRE].

[62] G.C. Dorsch, S.J. Huber and J.M. No, A strong electroweak phase transition in the 2HDM after LHC8, JHEP 10 (2013) 029 [arXiv: 1305.6610] [INSPIRE].

[63] G.C. Dorsch, S.J. Huber, K. Mimasu and J.M. No, Echoes of the Electroweak Phase Transition: Discovering a second Higgs doublet through $A_{0} \rightarrow Z H_{0}$, Phys. Rev. Lett. 113 (2014) 211802 [arXiv:1405.5537] [INSPIRE].

[64] C.P.D. Harman and S.J. Huber, Does zero temperature decide on the nature of the electroweak phase transition?, JHEP 06 (2016) 005 [arXiv: 1512.05611] [INSPIRE].

[65] P. Basler, M. Krause, M. Muhlleitner, J. Wittbrodt and A. Wlotzka, Strong First Order Electroweak Phase Transition in the CP-Conserving 2HDM Revisited, JHEP 02 (2017) 121 [arXiv: 1612.04086] [INSPIRE].

[66] G.C. Dorsch, S.J. Huber, K. Mimasu and J.M. No, The Higgs Vacuum Uplifted: Revisiting the Electroweak Phase Transition with a Second Higgs Doublet, JHEP 12 (2017) 086 [arXiv: 1705.09186] [INSPIRE].

[67] J. Bernon, L. Bian and Y. Jiang, A new insight into the phase transition in the early Universe with two Higgs doublets, JHEP 05 (2018) 151 [arXiv:1712.08430] [INSPIRE].

[68] J.O. Andersen, T. Gorda, A. Helset, L. Niemi, T.V.I. Tenkanen, A. Tranberg et al., Nonperturbative Analysis of the Electroweak Phase Transition in the Two Higgs Doublet Model, Phys. Rev. Lett. 121 (2018) 191802 [arXiv:1711.09849] [InSPIRE].

[69] K. Kainulainen, V. Keus, L. Niemi, K. Rummukainen, T.V.I. Tenkanen and V. Vaskonen, On the validity of perturbative studies of the electroweak phase transition in the Two Higgs Doublet model, JHEP 06 (2019) 075 [arXiv: 1904.01329] [INSPIRE].

[70] R. Zhou and L. Bian, Baryon asymmetry and detectable Gravitational Waves from Electroweak phase transition, arXiv:2001.01237 [INSPIRE]. 
[71] M. Carena, M. Quirós and C.E.M. Wagner, Opening the window for electroweak baryogenesis, Phys. Lett. B 380 (1996) 81 [hep-ph/9603420] [INSPIRE].

[72] D. Delepine, J.M. Gerard, R. Gonzalez Felipe and J. Weyers, A Light stop and electroweak baryogenesis, Phys. Lett. B 386 (1996) 183 [hep-ph/9604440] [INSPIRE].

[73] J.M. Cline and K. Kainulainen, Supersymmetric electroweak phase transition: Beyond perturbation theory, Nucl. Phys. B 482 (1996) 73 [hep-ph/9605235] [INSPIRE].

[74] M. Laine and K. Rummukainen, The MSSM electroweak phase transition on the lattice, Nucl. Phys. B 535 (1998) 423 [hep-lat/9804019] [InSPIRE].

[75] M. Carena, G. Nardini, M. Quirós and C.E.M. Wagner, The Baryogenesis Window in the MSSM, Nucl. Phys. B 812 (2009) 243 [arXiv:0809.3760] [InSPIRE].

[76] T. Cohen, D.E. Morrissey and A. Pierce, Electroweak Baryogenesis and Higgs Signatures, Phys. Rev. D 86 (2012) 013009 [arXiv:1203.2924] [InSPIRE].

[77] M. Laine, G. Nardini and K. Rummukainen, Lattice study of an electroweak phase transition at $m_{h} \simeq 126 \mathrm{GeV}, J C A P 01$ (2013) 011 [arXiv:1211.7344] [INSPIRE].

[78] D. Curtin, P. Jaiswal and P. Meade, Excluding Electroweak Baryogenesis in the MSSM, JHEP 08 (2012) 005 [arXiv: 1203.2932] [INSPIRE].

[79] M. Carena, G. Nardini, M. Quirós and C.E.M. Wagner, MSSM Electroweak Baryogenesis and LHC Data, JHEP 02 (2013) 001 [arXiv:1207.6330] [INSPIRE].

[80] A. Katz, M. Perelstein, M.J. Ramsey-Musolf and P. Winslow, Stop-Catalyzed Baryogenesis Beyond the MSSM, Phys. Rev. D 92 (2015) 095019 [arXiv: 1509.02934] [INSPIRE].

[81] P. Fileviez Perez, H.H. Patel, M.J. Ramsey-Musolf and K. Wang, Triplet Scalars and Dark Matter at the LHC, Phys. Rev. D 79 (2009) 055024 [arXiv:0811.3957] [InSPIRE].

[82] T.A. Chowdhury, M. Nemevšek, G. Senjanović and Y. Zhang, Dark Matter as the Trigger of Strong Electroweak Phase Transition, JCAP 02 (2012) 029 [arXiv:1110.5334] [INSPIRE].

[83] H.H. Patel and M.J. Ramsey-Musolf, Stepping Into Electroweak Symmetry Breaking: Phase Transitions and Higgs Phenomenology, Phys. Rev. D 88 (2013) 035013 [arXiv:1212.5652] [INSPIRE].

[84] N. Blinov, J. Kozaczuk, D.E. Morrissey and C. Tamarit, Electroweak Baryogenesis from Exotic Electroweak Symmetry Breaking, Phys. Rev. D 92 (2015) 035012 [arXiv: 1504.05195] [INSPIRE].

[85] L. Niemi, H.H. Patel, M.J. Ramsey-Musolf, T.V.I. Tenkanen and D.J. Weir, Electroweak phase transition in the real triplet extension of the SM: Dimensional reduction, Phys. Rev. $D 100$ (2019) 035002 [arXiv: 1802.10500] [INSPIRE].

[86] W. Chao, G.-J. Ding, X.-G. He and M. Ramsey-Musolf, Scalar Electroweak Multiplet Dark Matter, JHEP 08 (2019) 058 [arXiv: 1812.07829] [INSPIRE].

[87] N.F. Bell, M.J. Dolan, L.S. Friedrich, M.J. Ramsey-Musolf and R.R. Volkas, Two-Step Electroweak Symmetry-Breaking: Theory Meets Experiment, JHEP 05 (2020) 050 [arXiv: 2001.05335] [INSPIRE].

[88] C.-W. Chiang, G. Cottin, Y. Du, K. Fuyuto and M.J. Ramsey-Musolf, Collider Probes of Real Triplet Scalar Dark Matter, JHEP 01 (2021) 198 [arXiv: 2003.07867] [INSPIRE].

[89] L. Niemi, M. Ramsey-Musolf, T.V.I. Tenkanen and D.J. Weir, Thermodynamics of a two-step electroweak phase transition, arXiv:2005.11332 [INSPIRE]. 
[90] M. Cirelli, N. Fornengo and A. Strumia, Minimal dark matter, Nucl. Phys. B 753 (2006) 178 [hep-ph/0512090] [INSPIRE].

[91] M. Cirelli, A. Strumia and M. Tamburini, Cosmology and Astrophysics of Minimal Dark Matter, Nucl. Phys. B 787 (2007) 152 [arXiv:0706.4071] [INSPIRE].

[92] M. Cirelli, R. Franceschini and A. Strumia, Minimal Dark Matter predictions for galactic positrons, anti-protons, photons, Nucl. Phys. B 800 (2008) 204 [arXiv:0802.3378] [INSPIRE].

[93] O. Fischer and J.J. van der Bij, Multi-singlet and singlet-triplet scalar dark matter, Mod. Phys. Lett. A 26 (2011) 2039 [InSPIRE].

[94] S.S. AbdusSalam and T.A. Chowdhury, Scalar Representations in the Light of Electroweak Phase Transition and Cold Dark Matter Phenomenology, JCAP 05 (2014) 026 [arXiv: 1310.8152$]$ [INSPIRE].

[95] O. Fischer and J.J. van der Bij, The scalar Singlet-Triplet Dark Matter Model, JCAP 01 (2014) 032 [arXiv: 1311.1077] [InSPIRE].

[96] S. Yaser Ayazi and S.M. Firouzabadi, Constraining Inert Triplet Dark Matter by the LHC and FermiLAT, JCAP 11 (2014) 005 [arXiv: 1408.0654] [INSPIRE].

[97] S. Yaser Ayazi and S.M. Firouzabadi, Footprint of Triplet Scalar Dark Matter in Direct, Indirect Search and Invisible Higgs Decay, Cogent Phys. 2 (2015) 1047559 [arXiv: 1501.06176] [INSPIRE].

[98] M. Chala, M. Ramos and M. Spannowsky, Gravitational wave and collider probes of a triplet Higgs sector with a low cutoff, Eur. Phys. J. C 79 (2019) 156 [arXiv:1812.01901] [INSPIRE].

[99] L. Wang and X.-F. Han, LHC diphoton and Z+photon Higgs signals in the Higgs triplet model with $Y=0$, JHEP 03 (2014) 010 [arXiv:1303.4490] [INSPIRE].

[100] N. Khan, Exploring the hyperchargeless Higgs triplet model up to the Planck scale, Eur. Phys. J. C 78 (2018) 341 [arXiv: 1610.03178] [INSPIRE].

[101] M. Chabab, M.C. Peyranère and L. Rahili, Probing the Higgs sector of $Y=0$ Higgs Triplet Model at LHC, Eur. Phys. J. C 78 (2018) 873 [arXiv: 1805. 00286] [INSPIRE].

[102] H. Georgi and M. Machacek, Doubly charged Higgs bosons, Nucl. Phys. B 262 (1985) 463 [INSPIRE].

[103] M.S. Chanowitz and M. Golden, Higgs Boson Triplets With $M_{W}=M_{Z} \cos \theta_{\omega}$, Phys. Lett. B 165 (1985) 105 [INSPIRE].

[104] R. Zhou, W. Cheng, X. Deng, L. Bian and Y. Wu, Electroweak phase transition and Higgs phenomenology in the Georgi-Machacek model, JHEP 01 (2019) 216 [arXiv:1812.06217] [INSPIRE].

[105] L. Bian, H.-K. Guo, Y. Wu and R. Zhou, Gravitational wave and collider searches for electroweak symmetry breaking patterns, Phys. Rev. D 101 (2020) 035011 [arXiv: 1906.11664] [INSPIRE].

[106] P. Bandyopadhyay, C. Corianò and A. Costantini, Perspectives on a supersymmetric extension of the standard model with a $Y=0$ Higgs triplet and a singlet at the LHC, JHEP 09 (2015) 045 [arXiv: 1506.03634] [INSPIRE].

[107] S.A.R. Ellis, T. Gherghetta, K. Kaneta and K.A. Olive, New Weak-Scale Physics from SO(10) with High-Scale Supersymmetry, Phys. Rev. D 98 (2018) 055009 [arXiv: 1807.06488] [INSPIRE]. 
[108] S. Inoue, G. Ovanesyan and M.J. Ramsey-Musolf, Two-Step Electroweak Baryogenesis, Phys. Rev. D 93 (2016) 015013 [arXiv: 1508.05404] [InSPIRE].

[109] A. Dutta Banik, R. Roshan and A. Sil, Two Component Singlet-Triplet Scalar Dark Matter and Electroweak Vacuum Stability, arXiv:2009.01262 [INSPIRE].

[110] GAMBIT collaboration, Status of the scalar singlet dark matter model, Eur. Phys. J. C 77 (2017) 568 [arXiv: 1705.07931] [INSPIRE].

[111] G. Arcadi, A. Djouadi and M. Raidal, Dark Matter through the Higgs portal, Phys. Rept. 842 (2020) 1 [arXiv: 1903.03616] [INSPIRE].

[112] Particle Data Group collaboration, Review of Particle Physics, PTEP 2020 (2020) 083C01 [INSPIRE].

[113] J.R. Forshaw, D.A. Ross and B.E. White, Higgs mass bounds in a triplet model, JHEP 10 (2001) 007 [hep-ph/0107232] [INSPIRE].

[114] K. Kannike, Vacuum Stability of a General Scalar Potential of a Few Fields, Eur. Phys. J. C 76 (2016) 324 [Erratum ibid. 78 (2018) 355] [arXiv: 1603.02680] [INSPIRE].

[115] M. Gonderinger, H. Lim and M.J. Ramsey-Musolf, Complex Scalar Singlet Dark Matter: Vacuum Stability and Phenomenology, Phys. Rev. D 86 (2012) 043511 [arXiv:1202.1316] [INSPIRE].

[116] Y. Du, A. Dunbrack, M.J. Ramsey-Musolf and J.-H. Yu, Type-II Seesaw Scalar Triplet Model at a $100 \mathrm{TeV}$ pp Collider: Discovery and Higgs Portal Coupling Determination, JHEP 01 (2019) 101 [arXiv:1810.09450] [INSPIRE].

[117] G. Bélanger, F. Boudjema, A. Goudelis, A. Pukhov and B. Zaldivar, MicrOMEGAs5.0: Freeze-in, Comput. Phys. Commun. 231 (2018) 173 [arXiv:1801.03509] [InSPIRE].

[118] Planck collaboration, Planck 2018 results. VI. Cosmological parameters, Astron. Astrophys. 641 (2020) A6 [arXiv:1807.06209] [INSPIRE].

[119] XENON collaboration, Dark Matter Search Results from a One Ton-Year Exposure of XENON1T, Phys. Rev. Lett. 121 (2018) 111302 [arXiv:1805.12562] [INSPIRE].

[120] FERMI-LAT collaboration, Updated search for spectral lines from Galactic dark matter interactions with pass 8 data from the Fermi Large Area Telescope, Phys. Rev. D 91 (2015) 122002 [arXiv: 1506.00013] [INSPIRE].

[121] J. Alwall, R. Frederix, S. Frixione, V. Hirschi, F. Maltoni, O. Mattelaer et al., The automated computation of tree-level and next-to-leading order differential cross sections, and their matching to parton shower simulations, JHEP 07 (2014) 079 [arXiv: 1405.0301] [INSPIRE].

[122] W. Beenakker, M. Klasen, M. Krämer, T. Plehn, M. Spira and P.M. Zerwas, The Production of charginos/neutralinos and sleptons at hadron colliders, Phys. Rev. Lett. 83 (1999) 3780 [Erratum ibid. 100 (2008) 029901] [hep-ph/9906298] [INSPIRE].

[123] J. Alwall, C. Duhr, B. Fuks, O. Mattelaer, D.G. Öztürk and C.-H. Shen, Computing decay rates for new physics theories with FeynRules and MadGraph 5_aMC@NLO, Comput. Phys. Commun. 197 (2015) 312 [arXiv:1402.1178] [INSPIRE].

[124] CMS collaboration, Combined search for electroweak production of charginos and neutralinos in proton-proton collisions at $\sqrt{s}=13 \mathrm{TeV}$, JHEP 03 (2018) 160 [arXiv: 1801.03957] [INSPIRE]. 
[125] ATLAS collaboration, Search for chargino and neutralino production in final states with a Higgs boson and missing transverse momentum at $\sqrt{s}=13$ TeV with the ATLAS detector, Phys. Rev. D 100 (2019) 012006 [arXiv:1812.09432] [INSPIRE].

[126] ATLAS collaboration, Searches for electroweak production of supersymmetric particles with compressed mass spectra in $\sqrt{s}=13 \mathrm{TeV}$ pp collisions with the ATLAS detector, Phys. Rev. D 101 (2020) 052005 [arXiv:1911.12606] [INSPIRE].

[127] CMS collaboration, Search for supersymmetry with a compressed mass spectrum in the vector boson fusion topology with 1-lepton and 0-lepton final states in proton-proton collisions at $\sqrt{s}=13 \mathrm{TeV}$, JHEP 08 (2019) 150 [arXiv: 1905.13059] [INSPIRE].

[128] D. Dercks, N. Desai, J.S. Kim, K. Rolbiecki, J. Tattersall and T. Weber, CheckMATE 2: From the model to the limit, Comput. Phys. Commun. 221 (2017) 383 [arXiv:1611.09856] [INSPIRE].

[129] CMS collaboration, Search for supersymmetry using Higgs boson to diphoton decays at $\sqrt{s}=13 \mathrm{TeV}, \mathrm{JHEP} 11$ (2019) 109 [arXiv:1908.08500] [INSPIRE].

[130] T. Sjöstrand, S. Ask, J.R. Christiansen, R. Corke, N. Desai, P. Ilten et al., An introduction to PYTHIA 8.2, Comput. Phys. Commun. 191 (2015) 159 [arXiv:1410.3012] [INSPIRE].

[131] E. Conte and B. Fuks, Confronting new physics theories to LHC data with MADANALYSIS 5, Int. J. Mod. Phys. A 33 (2018) 1830027 [arXiv: 1808.00480] [INSPIRE].

[132] ATLAS collaboration, Searches for electroweak production of supersymmetric particles with compressed mass spectra in $\sqrt{s}=13$ TeV pp collisions with the ATLAS detector, figure 41ab (2020). https://doi.org/10.17182/hepdata.91374.v2/t79.

[133] ATLAS collaboration, Searches for electroweak production of supersymmetric particles with compressed mass spectra in $\sqrt{s}=13 \mathrm{TeV}$ pp collisions with the ATLAS detector, figure 41cd (2020). https://doi.org/10.17182/hepdata.91374.v2/t80.

[134] ATLAS collaboration, Search for direct production of electroweakinos in final states with missing transverse momentum and a Higgs boson decaying into photons in pp collisions at $\sqrt{s}=13$ TeV with the ATLAS detector, JHEP 10 (2020) 005 [arXiv:2004.10894] [INSPIRE].

[135] ATLAS collaboration, Search for chargino-neutralino production with mass splittings near the electroweak scale in three-lepton final states in $\sqrt{s}=13 \mathrm{TeV} p \mathrm{p}$ collisions with the ATLAS detector, Phys. Rev. D 101 (2020) 072001 [arXiv:1912.08479] [INSPIRE].

[136] ATLAS collaboration, Search for electroweak production of charginos and sleptons decaying into final states with two leptons and missing transverse momentum in $\sqrt{s}=13$ TeV pp collisions using the ATLAS detector, Eur. Phys. J. C 80 (2020) 123 [arXiv: 1908.08215] [INSPIRE].

[137] ATLAS collaboration, Search for electroweak production of charginos and sleptons decaying into final states with two leptons and missing transverse momentum in $\sqrt{s}=13$ TeV pp collisions using the ATLAS detector, xsec upper limits 1 (2019) https://doi.org/10.17182/hepdata.89413.v1/t45.

[138] S. Profumo, L. Ubaldi and C. Wainwright, Singlet Scalar Dark Matter: monochromatic gamma rays and metastable vacua, Phys. Rev. D 82 (2010) 123514 [arXiv:1009.5377] [INSPIRE].

[139] M. Duerr, P. Fileviez Pérez and J. Smirnov, Scalar Dark Matter: Direct vs. Indirect Detection, JHEP 06 (2016) 152 [arXiv: 1509.04282] [INSPIRE]. 